OPEN ACCESS

Edited by:

Yogesh Rathi,

Harvard Medical School, USA

Reviewed by:

Nicholas Bock

McMaster University, Canada Lipeng Ning,

Brigham and Women's Hospital, USA

${ }^{*}$ Correspondence:

Marcel Warntjes

marcel.warntjes@cmiv.liu.se

Specialty section:

This article was submitted to Brain Imaging Methods, a section of the journal Frontiers in Neurology

Received: 15 September 2015 Accepted: 02 February 2016

Published: 17 February 2016

Citation:

Warntjes M, Engström M, Tisell $A$ and Lundberg P (2016) Modeling the Presence of Myelin and Edema in the Brain Based on Multi-Parametric Quantitative MRI

Front. Neurol. 7:16. doi: 10.3389/fneur.2016.00016

\section{Modeling the Presence of Myelin and Edema in the Brain Based on Multi-Parametric Quantitative MRI}

\author{
Marcel Warntjes ${ }^{1,2 *}$, Maria Engström ${ }^{1,3}$, Anders Tisell $^{1,4}$ and Peter Lundberg ${ }^{1,4}$ \\ ${ }^{1}$ Center for Medical Image Science and Visualization (CMIV), Linköping University, Linköping, Sweden, ${ }^{2}$ Division of \\ Cardiovascular Medicine, Department of Medical and Health Sciences, Linköping University, Linköping, Sweden, ${ }^{3}$ Radiology, \\ Department of Medical and Health Sciences, Linköping University, Linköping, Sweden, ${ }^{4}$ Radiation Physics, Department of \\ Medical and Health Sciences, Linköping University, Linköping, Sweden
}

The aim of this study was to present a model that uses multi-parametric quantitative MRI to estimate the presence of myelin and edema in the brain. The model relates simultaneous measurement of $R_{1}$ and $R_{2}$ relaxation rates and proton density to four partial volume compartments, consisting of myelin partial volume, cellular partial volume, free water partial volume, and excess parenchymal water partial volume. The model parameters were obtained using spatially normalized brain images of a group of 20 healthy controls. The pathological brain was modeled in terms of the reduction of myelin content and presence of excess parenchymal water, which indicates the degree of edema. The method was tested on spatially normalized brain images of a group of 20 age-matched multiple sclerosis (MS) patients. Clear differences were observed with respect to the healthy controls: the MS group had a $79 \mathrm{~mL}$ smaller brain volume (1069 vs. $1148 \mathrm{~mL}$ ), a $38 \mathrm{~mL}$ smaller myelin volume (119 vs. $157 \mathrm{~mL}$ ), and a $21 \mathrm{~mL}$ larger excess parenchymal water volume (78 vs. $57 \mathrm{~mL}$ ). Template regions of interest of various brain structures indicated that the myelin partial volume in the MS group was $1.6 \pm 1.5 \%$ lower for gray matter (GM) structures and $2.8 \pm 1.0 \%$ lower for white matter (WM) structures. The excess parenchymal water partial volume was $9 \pm 10 \%$ larger for GM and $5 \pm 2 \%$ larger for WM. Manually placed ROls indicated that the results using the template ROls may have suffered from loss of anatomical detail due to the spatial normalization process. Examples of the application of the method on high-resolution images are provided for three individual subjects: a 45-year-old healthy subject, a 72-year-old healthy subject, and a 45-year-old MS patient. The observed results agreed with the expected behavior considering both age and disease. In conclusion, the proposed model may provide clinically important parameters, such as the total brain volume, degree of myelination, and degree of edema, based on a single qMRI acquisition with a clinically acceptable scan time.

Keywords: quantitative magnetic resonance imaging, brain tissue modeling, myelin, edema, $T_{1}$ relaxation, $T_{2}$ relaxation, proton density 


\section{INTRODUCTION}

Myelin is crucial for efficient signal transmission over long ranges in the nervous system because it increases the speed at which the impulses propagate along the axons. Axons are coated piecewise by multiple layers of phospholipid membranes ("sheaths") with embedded proteins produced by oligodendrocytes and Schwann cells in the central and peripheral nervous systems, respectively. Degradation of myelin impairs the signal transmission, and the nerve may eventually wither, leading to brain atrophy and brain dysfunction. Knowledge of myelin content supports the investigation of early brain development $(1,2)$. Accurate myelin measurements are valuable in studies of neurodegenerative diseases, such as multiple sclerosis (MS) $(3,4)$ and dementia $(5-7)$. Thus, measurements and monitoring of myelin content would provide important information for the diagnosis and prognosis in patients with suspected myelin degradation.

One established MRI method for myelin detection is based on the measurement of the multi-exponential transverse $\mathrm{T}_{2}$ relaxation time using a Carr-Purcell-Meiboom-Gill (CPMG) sequence $(8-10)$. The short-time component of the observed $\mathrm{T}_{2}$ relaxation represents the presence of water trapped between the myelin sheaths, termed myelin water $(\mathrm{MyW})$, whereas the medium-time $\mathrm{T}_{2}$ relaxation component is attributed to the intraand extracellular water. Commonly, the myelin water fraction (MWF), corresponding to the ratio of both components, is calculated. The proportionality of MWF with the myelin content has been verified in vitro and by histopathology $(11,12)$. More recently, an alternative approach called mcDESPOT was developed (13). This method consists of a combination of spoiled gradient echo (SPGR) and balanced steady-state free precession (bSSFP) acquisitions at multiple flip angles, resulting in the measurement of $\mathrm{MyW}$ and intra- and extracellular water pools. In particular, the mcDESPOT method has been applied to myelin development in children (14).

Limitations of the two described methods are mainly practical. Due to the very short myelin $\mathrm{T}_{2}$ relaxation time (10-15 ms), the multi-exponent $\mathrm{T}_{2}$ measurement mainly depends on the amplitude of the first echo signal, and mcDESPOT is highly sensitive to the accuracy of the applied flip angle, making the measurements demanding in terms of both SNR and time as well as highly dependent on corrections for $\mathrm{B}_{1}$ field and RF pulse profile effects. The underlying models of both approaches are considerably different, resulting in widespread estimations of the myelin content.

Here, we propose a model to estimate the presence of myelin and edema in the brain based on multi-parametric quantitative MRI (qMRI), where the longitudinal relaxation rate $R_{1}$, transverse relaxation rate $R_{2}$, and proton density $P D$ are determined simultaneously in one acquisition. It was previously reported that pathological processes, such as axonal damage, gliosis, inflammation, and edema are related to changes in the values of $R_{1}$, $\mathrm{R}_{2}$, and PD (15-19). Currently, multi-parametric MR quantification of $R_{1}, R_{2}$, and $P D$ can be achieved at high resolution within a 6-8 min scan time (20), which would make such an approach attractive for routine clinical use. The aim of this study was to present a model that relates the appearance of a qMRI-derived $\mathrm{R}_{1}-\mathrm{R}_{2}-\mathrm{PD}$ data structure to the myelin partial volume of the brain. The model parameters were derived based on data from Ref. (21), where brain images of a group of healthy controls were spatially normalized and averaged to characterize the healthy brain. The second aim of this study was to explore the possibilities of the model to detect both the differences in myelin content and the presence of edema in the pathological brain. Examples of the application of the method are provided for a group of MS patients and three individual subjects.

\section{MATERIALS AND METHODS}

\section{The Relaxation Model}

The proposed model for the observed $R_{1}, R_{2}$, and $P D$ values of the brain is visualized in Figure 1: each MRI acquisition voxel is composed of four partial volume compartments: the myelin partial volume $V_{M Y}$, cellular partial volume $V_{C L}$, free water partial volume $\mathrm{V}_{\mathrm{FW}}$, and excess parenchymal water partial volume $\mathrm{V}_{\mathrm{EPW}}$. The content in each partial volume compartment can range from 0 to $100 \%$, where the sum of the four compartments is $100 \%$. Each partial volume compartment has its own relaxation properties $\left(R_{1, M Y}, R_{2, M Y}, P D_{M Y}\right.$, $\mathrm{R}_{1, \mathrm{CL}}, \mathrm{R}_{2, \mathrm{CL}}, \mathrm{PD}_{\mathrm{CL}}, \mathrm{R}_{1, \mathrm{FW}}, \mathrm{R}_{2, \mathrm{FW}}, \mathrm{PD}_{\mathrm{FW}}, \mathrm{R}_{1, \mathrm{EPW}}, \mathrm{R}_{2, \mathrm{EPW}}, \mathrm{PD}_{\mathrm{EPW}}$ ), without further detailed knowledge of the multitude of interacting pools within each of the compartments. Using this approach, each partial volume compartment can be described by its $\mathrm{R}_{1}-\mathrm{R}_{2}-\mathrm{PD}$ values, its fraction of the acquisition voxel and the magnetization exchange with other partial volume compartments. The total acquisition voxel exhibits $R_{1}-R_{2}-P D$ values that reflect the effective, combined relaxation behavior of all four compartments. An MR quantification sequence measures the effective $\mathrm{R}_{1}-\mathrm{R}_{2}-\mathrm{PD}$ values of acquisition voxels in the total imaging volume, which can provide input to the model.

The $\mathrm{V}_{\mathrm{MY}}$ contains the thin layers of $\mathrm{MyW}$ and myelin sheets that are closely packed around the axons. The close proximity of $\mathrm{MyW}$ to the surrounding structure results in a very fast relaxation of this compartment. The $\mathrm{V}_{\mathrm{CL}}$ consists of intraand extracellular (interstitial) water, axonal water, and all cellular macromolecules, not being related to myelin. The presence of the macromolecules results in a medium-time relaxation of $\mathrm{V}_{\mathrm{CL}}$, which is slower than $\mathrm{V}_{\mathrm{MY}}$, but longer than $\mathrm{V}_{\mathrm{FW}}$. Between $V_{M Y}$ and $V_{C L}$, there is a magnetization exchange rate $k_{\mathrm{VMY}-\mathrm{VCL}}$. In the model, acquisition voxels in the normal brain parenchyma contain a mixture of $\mathrm{V}_{\mathrm{MY}}$ and $\mathrm{V}_{\mathrm{CL}}$, where voxels in gray matter (GM) have a low $V_{M Y}$ and voxels in white matter (WM) have a high $\mathrm{V}_{\mathrm{MY}}$ (see Figure 1A). The two compartments $\mathrm{V}_{\mathrm{MY}}$ and $\mathrm{V}_{\mathrm{CL}}$ are an approximation of the fourpool model (22), where $\mathrm{V}_{\mathrm{MY}}$ contains MyW and myelin semisolids and $\mathrm{V}_{\mathrm{CL}}$ contains intracellular and extracellular water and non-myelin semi-solids pools, albeit with less degrees of freedom. 


\section{Acquisition Voxel}
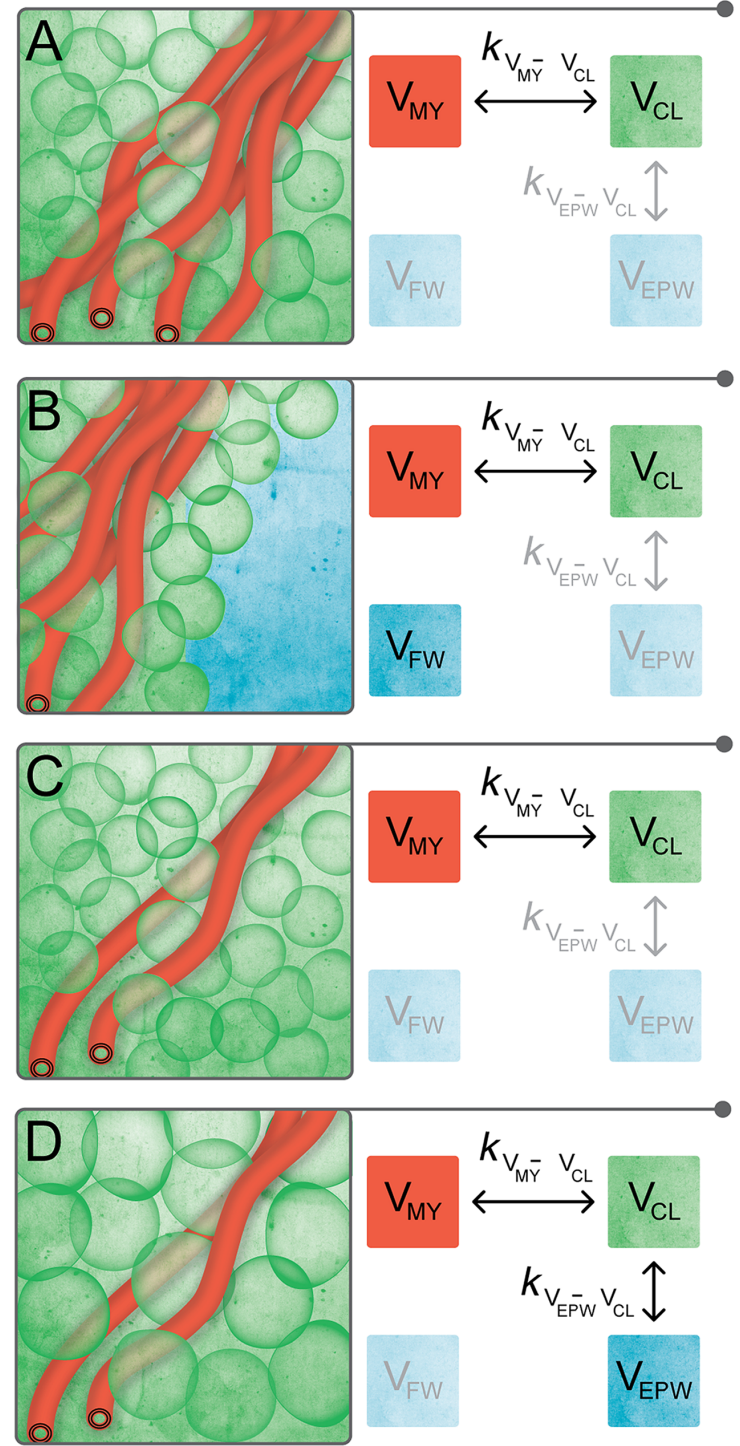

FIGURE 1 | Proposed compartmental exchange system for modeling brain parenchyma. Each MRI acquisition voxel is composed of four partial volume compartments, where each partial volume can range from 0 to $100 \%$, and where the sum is $100 \%$. A compartment is grayed out when its partial volume is equal to 0 . (A) Normal brain parenchyma consists of myelin partial volume $V_{M Y}$ and cellular partial volume $V_{C L}$. Between $V_{M Y}$ and $V_{C L}$, there is a magnetization net exchange rate $k_{V M Y}-V_{C L}$. (B) At the interface of brain parenchyma with the surrounding bulk CSF, an acquisition voxel contains a mixture of $V_{M Y}$ and $V_{C L}$ (i.e., brain parenchyma) and free water partial volume $V_{F W}$. There is no magnetization exchange between $V_{F W}$ and the other partial volumes. (C) In pathological brain parenchyma, myelin loss may occur, resulting in a relative decrease in $\mathrm{V}_{\mathrm{MY}}$. The relative amount of $\mathrm{V}_{\mathrm{CL}}$ in the acquisition voxel increases to maintain 100\% tissue, resulting in a decrease in the total brain volume. (D) Alternatively, there can be edema in pathological brain parenchyma, included in the model by the presence of the non-zero excess parenchymal water partial volume $V_{E P W}$. No distinction can be made between excess parenchymal water and the already present parenchymal water of the $V_{C L}$, making the exchange rate $k_{V E P W-V C L}$ infinitely high. The combination of $V_{C L}$ and $V_{E P W}$ effectively dilutes the myelin content, resulting in a relative decrease in $V_{\mathrm{MY}}$ per acquisition voxel and an increase in the total brain volume.
The brain is surrounded by cerebrospinal fluid (CSF), making it necessary to add a free water partial volume $\mathrm{V}_{\mathrm{FW}}$ to the model, as also pointed out in Ref. (23). Because bulk CSF is physically separated from the brain parenchyma except for the interface, there is no magnetization exchange between $V_{\mathrm{FW}}$ and any other compartment (i.e. "free"). Hence, at the border of the brain, acquisition voxels contain a mixture of $\mathrm{V}_{\mathrm{MY}}$ and $\mathrm{V}_{\mathrm{CL}}$ (brain parenchyma) and $\mathrm{V}_{\mathrm{FW}}$ (CSF), see Figure 1B.

In the pathological brain, two distinct processes are modeled: compared with the normal brain, there can be myelin loss, resulting in a relative decrease in $\mathrm{V}_{\mathrm{MY}}$. To maintain $100 \%$ tissue, the relative amount of $\mathrm{V}_{\mathrm{CL}}$ in an acquisition voxel will increase. Therefore, the loss of myelin results in a compaction of the brain and, thus, a decrease in the total brain volume (Figure 1C). The second process is the occurrence of edema, modeled as the presence of excess parenchymal water partial volume $V_{E P W}$, which adds water to $V_{C L}$. No distinction can be made between excess parenchymal water and the already present parenchymal water of $\mathrm{V}_{\mathrm{CL}}$ and, therefore, the exchange rate $k_{\mathrm{VEPW}-\mathrm{VCL}}$ is infinitely high. Modeling two separate partial volume compartments with an infinite exchange is a mathematical approach to acquire knowledge on the degree of edema without knowledge of the exact internal composition of $\mathrm{V}_{\mathrm{CL}}$. The cellular swelling due to a non-zero $\mathrm{V}_{\mathrm{EPW}}$ effectively dilutes the myelin present in the acquisition voxel, resulting in a relative decrease in $\mathrm{V}_{\mathrm{MY}}$. In this case, the total brain volume increases (Figure 1D).

\section{Bloch Simulation}

A numerical simulation of coupled Bloch equations of the four partial volume compartments was performed using 150 identical magnetization elements $i$, spread equidistantly over a distance of $15 \mathrm{~mm}$ in the acquisition slice direction, where each element had a distance $d_{\mathrm{i}}$ from the center of the slice. Each of the 150 elements consisted of the same partial volume distribution of interacting $\mathrm{V}_{\mathrm{MY}}, \mathrm{V}_{\mathrm{CL}}, \mathrm{V}_{\mathrm{FW}}$, and $\mathrm{V}_{\mathrm{EPW}}$ with normalized magnetization vectors $M_{\mathrm{MY}}, M_{\mathrm{CL}}, M_{\mathrm{FW}}$, and $M_{\mathrm{EPW}}$, respectively. The evolution of each magnetization $M_{\mathrm{i}}=\left[\begin{array}{lll}M_{\mathrm{x}} & M_{\mathrm{y}} & M_{\mathrm{z}}\end{array}\right]_{i}^{\mathrm{T}}$ was calculated in small time-steps $t$, where each sequential magnetization $M_{\mathrm{i}, \mathrm{n}+1}$ of each element $i$ was calculated from the original magnetization $M_{\mathrm{i}, \mathrm{n}}$ using:

$$
M_{i, n+1}=R_{R F} * R_{G R} * R_{R 1} * R_{R 2} * M_{i, n}
$$

$R_{\mathrm{RF}}$ is the rotation matrix for the applied slice-selective RF pulses. The envelope of the RF pulses was approximated by a series of block pulses with constant amplitudes over the time interval $t$. The rotation flip angle $\alpha$, achieved in time $t$ over the $\mathrm{x}$ - or $\mathrm{y}$-axis, is equal to $2 \pi \gamma B_{1} t$, where $B_{1}$ is the amplitude of the RF pulse at that particular time interval, and $\gamma$ is the gyromagnetic ratio. $R_{\mathrm{GR}}$ is the rotation matrix for the applied slice-selective gradients. The rotation flip angle $\omega$, achieved in time $t$ over the $z$-axis, is equal to $2 \pi \gamma G d_{i} t$, where $G$ is the gradient strength and $d_{\mathrm{i}}$ is the distance from the center of the slice.

$R_{\mathrm{R} 1}$ is the relaxation matrix for the elements for the longitudinal relaxation rate $\mathrm{R}_{1} \cdot \boldsymbol{R}_{\mathrm{R} 1}$ only acts on the $M_{\mathrm{z}}$ component of each $M_{\mathrm{i}}$ according to: 


$$
\left[\begin{array}{c}
M_{z, M Y} \\
M_{z, C L} \\
M_{z, F W} \\
M_{z, E P W}
\end{array}\right]_{i, n+1}=\left[\begin{array}{cccc}
E_{1, M Y}-S_{M Y}\left(1-K_{M C}\right) & S_{M Y}\left(1-K_{M C}\right) & 0 & 0 \\
S_{C L a}\left(1-K_{M C}\right) & E_{1, C L}-S_{C L a}\left(1-K_{M C}\right)-S_{C L b} & 0 & S_{C L b} \\
0 & 0 & E_{1, F W} & 0 \\
0 & S_{E P W} & 0 & E_{1, E P W}-S_{E P W}
\end{array}\right] *\left[\begin{array}{c}
M_{z, M Y} \\
M_{z, C L} \\
M_{z, F W} \\
M_{z, E P W}
\end{array}\right]_{i, n}+\left[\begin{array}{c}
1-E_{1, M Y} \\
1-E_{1, C L} \\
1-E_{1, F W} \\
1-E_{1, E P W}
\end{array}\right]
$$

where $\quad E_{1, \mathrm{MY}}=\exp \left(-\mathrm{tR}_{1, \mathrm{MY}}\right), \quad E_{1, \mathrm{CL}}=\exp \left(-\mathrm{tR}_{1, \mathrm{CL}}\right), \quad E_{1, \mathrm{FW}}=$ $\exp \left(-\mathrm{tR}_{1, \mathrm{FW}}\right), E_{1, \mathrm{EPW}}=\exp \left(-\mathrm{tR}_{1, \mathrm{EPW}}\right)$ and $K_{M C}=\exp \left(-\mathrm{t} k_{\mathrm{MY}-\mathrm{CL}}\right)$. The exchange rate $K_{M C}$ is the combined forward and backward exchange rate between $\mathrm{V}_{\mathrm{MY}}$ and $\mathrm{V}_{\mathrm{CL}}$. The exchange rate between $\mathrm{V}_{\mathrm{EPW}}$ and $\mathrm{V}_{\mathrm{CL}}$ is infinitely high. The scaling factors $S_{\mathrm{MY}}=$ $\mathrm{V}_{\mathrm{CL}} * \mathrm{PD}_{\mathrm{CL}} /\left(\mathrm{V}_{\mathrm{MY}} * \mathrm{PD}_{\mathrm{MY}}+\mathrm{V}_{\mathrm{CL}}\right.$ * $\left.\mathrm{PD}_{\mathrm{CL}}\right), S_{\mathrm{CLa}}=\mathrm{V}_{\mathrm{MY}} * \mathrm{PD}_{\mathrm{MY}} /$ $\left(\mathrm{V}_{\mathrm{MY}}{ }^{*} \mathrm{PD}_{\mathrm{MY}}+\mathrm{V}_{\mathrm{CL}}{ }^{*} \mathrm{PD}_{\mathrm{CL}}\right), \quad S_{\mathrm{CLb}}=\mathrm{V}_{\mathrm{EPW}}{ }^{*} \mathrm{PD}_{\mathrm{EPW}} /\left(\mathrm{V}_{\mathrm{EPW}}{ }^{*}\right.$ $\left.\mathrm{PD}_{\mathrm{EPW}}+\mathrm{V}_{\mathrm{CL}}{ }^{*} \mathrm{PD}_{\mathrm{CL}}\right)$ and $S_{\mathrm{EPW}}=\mathrm{V}_{\mathrm{CL}}{ }^{*} \mathrm{PD}_{\mathrm{CL}} /\left(\mathrm{V}_{\mathrm{EPW}}{ }^{*}\right.$ $\left.\mathrm{PD}_{\mathrm{EPW}}+\mathrm{V}_{\mathrm{CL}}{ }^{*} \mathrm{PD}_{\mathrm{CL}}\right)$ are required to take the relative volumes of $\mathrm{PD}$ in each compartment into account.

$R_{\mathrm{R} 2}$ is the relaxation matrix for the elements for the transverse relaxation rate $\mathrm{R}_{2} \cdot R_{\mathrm{R} 2}$ only acts on the $M_{\mathrm{xy}}$ component of each $M_{i}$ according to: of $180^{\circ}$ over the $y$-axis. The refocusing pulses were straddled by spoiler gradients. The scan time was 8:21 min on a Philips Achieva 1.5T (Philips Healthcare, Best, The Netherlands).

\section{Application of the Bloch Simulation on the Quantification Sequence}

The RF pulses, gradients, and timings of the quantification sequence were implemented as a script into the model calculations. The product of all matrices in Eq. 1 does not commute $(\mathrm{AB} \neq \mathrm{BA})$ and, therefore, Eq. 1 is only valid if time-steps are chosen such that the relaxation rates cause a near-zero change of magnetization per time step. Typical relaxation in the brain occurs

$$
\left[\begin{array}{c}
M_{x y, M Y} \\
M_{x y, C L} \\
M_{x y, F W} \\
M_{x y, E P W}
\end{array}\right]_{i, n+1}=\left[\begin{array}{cccc}
E_{2, M Y}-S_{M Y}\left(1-K_{M C}\right) & S_{M Y}\left(1-K_{M C}\right) & 0 & 0 \\
S_{C L a}\left(1-K_{M C}\right) & E_{2, C L}-S_{C L a}\left(1-K_{M C}\right)-S_{C L b} & 0 & S_{C L b} \\
0 & 0 & E_{2, F W} & 0 \\
0 & S_{E P W} & 0 & E_{2, E P W}-S_{E P W}
\end{array}\right] *\left[\begin{array}{c}
M_{x y, M Y} \\
M_{x y, C L} \\
M_{x y, F W} \\
M_{x y, E P W}
\end{array}\right]_{i, n}
$$

where $\quad E_{2, \mathrm{MY}}=\exp \left(-\mathrm{tR}_{2, \mathrm{MY}}\right), \quad E_{2, \mathrm{Cl}}=\exp \left(-\mathrm{tR}_{2, \mathrm{CL}}\right), \quad E_{2, \mathrm{FW}}=$ $\exp \left(-\mathrm{tR}_{2, \mathrm{FW}}\right), E_{2, \mathrm{ECB}}=\exp \left(-\mathrm{tR}_{2, \mathrm{ECB}}\right)$.

\section{MR Quantification Sequence}

The presented Bloch equations form a general description of the magnetization evolution for each acquisition voxel and only have meaning when applied to an actual MRI sequence. The specifics of this MRI sequence, with the applied RF pulses, gradients, and timings, dictate the observable signal behavior. The MRI quantification method employed was a multi-echo, multidelay saturation recovery spin echo sequence (QRAPMASTER) as described previously (20). It was a multi-slice sequence where slice-selective saturation pulses were interleaved with a CPMG acquisition of 5 echoes at 14-ms multiples. The saturation pulse acted on slice $n$, whereas the subsequent acquisition acted on slice $m$. By a fixed shift between slices $n$ and $m$, an effective delay time TD was created between the saturation and acquisition of each particular slice. The sequence was repeated four times where the shift between $n$ and $m$, and hence the saturation delay, was changed. The result of the sequence was a matrix of 20 images at five different echo times TE and at four different saturation delay times TD. The applied slice-selective RF pulse profiles and amplitudes, gradient strengths, and timings were extracted from the scanner. The repetition time TR was $2950 \mathrm{~ms}$ with 30 slices of 4$\mathrm{mm}$ thickness with an in-plane resolution of $1 \mathrm{~mm}$. The saturation pulse had a flip angle of $120^{\circ}$ over the $x$-axis followed by a delay of $100,400,1380$, and $2860 \mathrm{~ms}$, corresponding to a shift between $n$ and $m$ of $1,4,14$, and 29 slices, respectively. The excitation pulse had a flip angle of $90^{\circ}$ over the $\mathrm{x}$-axis, followed by refocusing pulses in the order of millisecond. Therefore, we choose time steps $t$ of $1 \mu \mathrm{s}$, which is three orders of magnitude smaller, but still results in a reasonable calculation time. The observable signal intensity $I$ at each combination of TE and TD was calculated as the product of the total proton density for each partial volume $\left(\mathrm{V}^{\star} \mathrm{PD}\right)$ and the $x y$-component of the magnetization $M_{\mathrm{i}}$ of these spins, summed over all elements i:

$$
\begin{aligned}
I_{T E, T D}= & \sum_{i}\left(V_{M Y} * P D_{M Y} * M_{x y, M Y}+V_{C L} * P D_{C L} * M_{x y, C L}\right. \\
& +V_{F W} * P D_{F W} * M_{x y, F W} \\
& \left.+V_{E P W} * P D_{E P W} * M_{x y, E P W}\right)_{T E, T D}
\end{aligned}
$$

In this way, the Block simulation also produced 20 images with different TE and TD, identical to the in vivo quantification sequence.

\section{Subjects}

MR quantification was performed on two groups of subjects, one with 20 patients diagnosed with Clinically Definite Multiple Sclerosis ( 5 males and 15 females; mean age of $47 \pm 12$ years). The mean extended disability status scale [EDSS (24)] of the MS group was $3.6 \pm 2.2$, and the mean disease duration was $15 \pm 10$ years. The second group consisted of age- and gender-matched healthy controls (5 males and 15 females; mean age of $47 \pm 11$ years). Three female participants were used as individual examples: one healthy subject of 45 years old, one healthy subject of 72 years old, and a secondary progressive MS patient of 45 years old (EDSS of 3.5; disease duration of 17 years). The study was approved by the regional ethical review board and written informed consent was 
obtained from all participants (full name of the board: "Regionala etikprövningsnämnden i Linköping"; registered under number Dnr. M88-07).

\section{Image Post-Processing}

$\mathrm{R}_{1}, \mathrm{R}_{2}$, and $\mathrm{PD}$ maps were retrieved from both the simulated and in vivo acquired images using SyMRI 7.0 (SyntheticMR, Linköping, Sweden). In summary, a least squares fit was performed as a function of the different TE and TD times according to:

$$
\begin{aligned}
& I_{T E, T D}=A \cdot P D \cdot \exp \left(-R_{2} T E\right) \\
& \times \frac{1-\left[1-\cos \left(B_{1} \theta\right)\right] \cdot \exp \left(-R_{1} T D\right)-\cos \left(B_{1} \theta\right) \cdot \exp \left(-R_{1} T R\right)}{1-\cos \left(B_{1} \alpha\right) \cdot \cos \left(B_{1} \theta\right) \cdot \exp \left(-R_{1} T R\right)}
\end{aligned}
$$

where $\alpha$ is the excitation flip angle, $\theta$ is the saturation flip angle, and $B_{1}$ is the amplitude of the $B_{1}$ field. $A$ is an overall scaling factor that considers the coil sensitivity, RF chain amplification, and voxel volume (20). This equation explicitly has two monoexponential functions, in $\mathrm{R}_{1}$ and $\mathrm{R}_{2}$, and hence it will reflect the dominant component of the relaxation behavior.

For spatial normalization of the in vivo brain data, the $\mathrm{R}_{1}, \mathrm{R}_{2}$, and PD maps were used to synthesize a stack of $\mathrm{T}_{2}$-weighted images with $\mathrm{TE}=100 \mathrm{~ms}$ and $\mathrm{TR}=4500 \mathrm{~ms}$. The synthetic $\mathrm{T}_{2}$ weighted images were smoothed with an 8 -mm Gaussian kernel and used as source images to calculate the transformation matrix to a standard stereotactic space in Montreal Neurological Institute (MNI) coordinates (26). The images were then transformed to match the size and position of a standard template using a 12-parameter (translation, rotation, shear, zoom) affine regularization and non-linear deformations by a linear combination of three-dimensional discrete cosine basis functions. The same transformation matrix was then applied to the $\mathrm{R}_{1}, \mathrm{R}_{2}$, and $\mathrm{PD}$ maps. The resulting data were re-gridded to $2 \mathrm{~mm} \times 2 \mathrm{~mm} \times 2 \mathrm{~mm}$ to obtain an isotropic dataset. All of the subjects were averaged to obtain the mean $R_{1}-R_{2}-P D$ values of the $\mathrm{MS}$ and control group. Finally, the mean $\mathrm{R}_{1}, \mathrm{R}_{2}$, and $\mathrm{PD}$ values were used as coordinates in a $\mathrm{R}_{1}-\mathrm{R}_{2}-\mathrm{PD}$ multi-parametric space, as presented in Ref. (21). The $2 \mathrm{D}$ histograms of the entire brain were created with 200 bins for $R_{1}$ on a scale of $0-2 s^{-1}, 200$ bins for $\mathrm{R}_{2}$ on a scale of $0-15 \mathrm{~s}^{-1}$, and 200 bins for PD on a scale of $50-100 \%$.

\section{Determining the Model Parameters}

The procedure to determine the model parameters is schematically depicted in Figure 2. In the model, the relaxation parameters for water, both for $\mathrm{V}_{\mathrm{FW}}$ and $\mathrm{V}_{\mathrm{EPW}}$, were fixed to literature values for CSF at $\mathrm{R}_{1}=0.24 \mathrm{~s}^{-1}, \mathrm{R}_{2}=0.87 \mathrm{~s}^{-1}$, and $\mathrm{PD}=100 \%$ (20). Additionally, the $\mathrm{R}_{2}$ relaxation for $\mathrm{V}_{\mathrm{MY}}$ was fixed to a reported value, at $R_{2, M Y}=77 \mathrm{~s}^{-1}$ (corresponding to $T_{2, \mathrm{MY}}=13 \mathrm{~ms}$ ) (22). Therefore, only six remaining model parameters, $\mathrm{R}_{1, \mathrm{MY}}, \mathrm{PD}_{\mathrm{MY}}$, $\mathrm{R}_{1, \mathrm{CL}}, \mathrm{R}_{2, \mathrm{CL}}, \mathrm{PD}_{\mathrm{CL}}$, and $k_{\mathrm{MY}-\mathrm{CL}}$, were allowed to vary. The six model parameters were given a random value under the restriction that $\mathrm{R}_{1, \mathrm{FW}}<\mathrm{R}_{1, \mathrm{CL}}<\mathrm{R}_{1, \mathrm{MY}}$ and $\mathrm{R}_{2, \mathrm{FW}}<\mathrm{R}_{2, \mathrm{CL}}<\mathrm{R}_{2, \mathrm{MY}}$. For each set of variable parameters, the magnetization evolution was calculated for all combinations of $\mathrm{V}_{\mathrm{MY}}$ and $\mathrm{V}_{\mathrm{CL}}$ and for all combinations

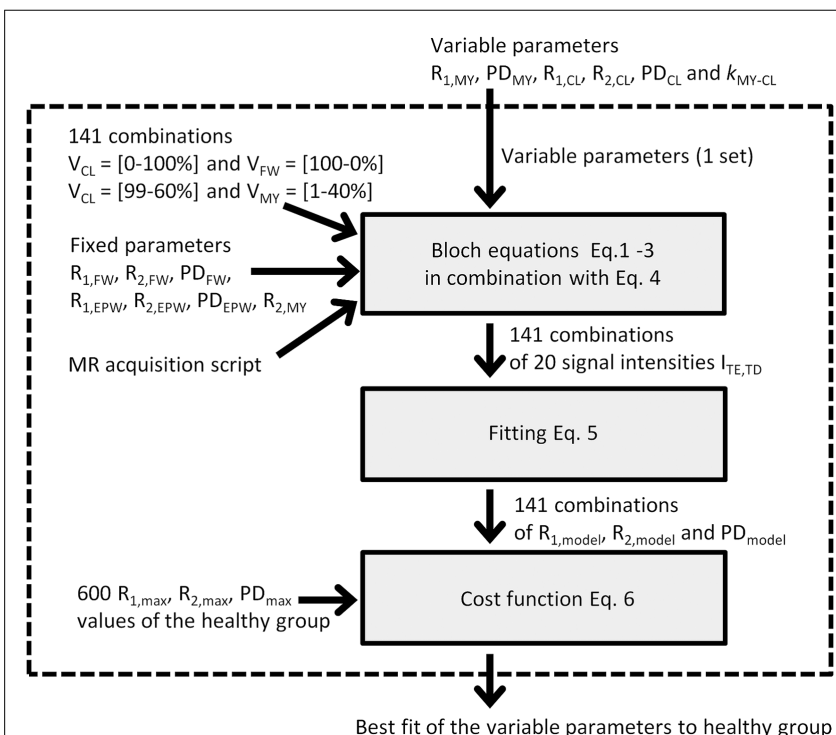

FIGURE 2 |Schematic depiction of the procedure to optimize the variable parameters: One set of variable parameters is chosen and evaluated within the dotted box. Evaluation is performed by running the Bloch equations of the simulated MR acquisition on 141 combinations of $V_{M Y}$, $V_{C L}$, and $V_{F W}$. This provides 20 signal intensities at various echo times and saturation delays times. The 20 signal intensities are fitted, resulting in an $R_{1}$, $R_{2}$, and $P D$ value of the model. The model values are then compared to the observed $R_{1}, R_{2}$, and $P D$ values of the healthy group using the maximum values in the $2 \mathrm{D}$ histograms. A cost function provides a measure for closeness of the model $R_{1}, R_{2}$, and PD values to the observed $R_{1}, R_{2}$, and $P D$ values. The evaluation is performed for many sets of variable parameters, resulting in the best fit.

of $\mathrm{V}_{\mathrm{CL}}$ and $\mathrm{V}_{\mathrm{FW}}$, using steps of $1 \%$ partial volume. Since the maximum amount is $100 \%$, a setting of for example $20 \% \mathrm{~V}_{\mathrm{FW}}$ requires a setting of $80 \% \mathrm{~V}_{\mathrm{CL}}$, hence producing 101 combinations of $\mathrm{V}_{\mathrm{FW}}$ and $\mathrm{V}_{\mathrm{CL}}$. $\mathrm{V}_{\mathrm{MY}}$ was restricted to a maximum of $40 \%$, since no higher values were expected to occur in the brain and we wanted to avoid values that could not be evaluated. This produced 40 combinations of $\mathrm{V}_{\mathrm{MY}}$ and $\mathrm{V}_{\mathrm{CL}}$, making a total of 141 combinations. The magnetization evolution was calculated using Eqs 1-3, resulting in the signal intensities $\mathrm{I}_{\mathrm{TE}, \mathrm{TD}}$ at five different echo times $\mathrm{TE}$ and four different saturation delay times TD for each partial volume combination (Eq. 4). The sets of $20 \mathrm{I}_{\mathrm{TE}, \mathrm{TD}}$ values were then fitted using Eq. 5, resulting in $141 \mathrm{R}_{1 \text {,model }}, \mathrm{R}_{2 \text {,model }}$, and $\mathrm{PD}_{\text {model }}$ values for each specific set of variable parameters.

To evaluate how close these $141 \mathrm{R}_{1}-\mathrm{R}_{2}-\mathrm{PD}$ values mimicked the observed data structure in the $2 \mathrm{D}$ histograms of the healthy control group, the maximum values in the histogram for each bin in $R_{1}$ were determined, and the corresponding $R_{2}$ and $P D$ values were recorded. This procedure was repeated for $R_{2}$ and PD. Because the $2 \mathrm{D}$ histograms had $200 \times 200$ bins, this procedure provided $600 \mathrm{R}_{1, \max }, \mathrm{R}_{2 \text {,max }}$, and $\mathrm{PD}_{\max }$ values to define the characteristic data structure of the healthy group. From these 600 combinations, 141 were selected that were closest to the 141 model combinations.

Finally, a cost function was set up to evaluate the difference between the $R_{1 \text {,model }}, R_{2 \text {,model, }}$ and $\mathrm{PD}_{\text {model }}$ values for each 
parameter setting with the selected $\mathrm{R}_{1, \max }, \mathrm{R}_{2 \text {,max }}$, and $\mathrm{PD}_{\max }$ values of the $2 \mathrm{D}$ histograms of the in vivo spatially normalized data:

$$
\begin{aligned}
f_{\text {cost }}= & \frac{1}{n} \sum\left[\left(\frac{R_{2, \text { model }}-R_{2, \text { max }}}{\sigma\left(R_{2}\right)}\right)^{2}+\left(\frac{P D_{\text {model }}-P D_{\text {max }}}{\sigma(P D)}\right)^{2}\right]_{R_{1}} \\
& +\left[\left(\frac{R_{1, \text { model }}-R_{1, \text { max }}}{\sigma\left(R_{1}\right)}\right)^{2}+\left(\frac{P D_{\text {model }}-P D_{\text {max }}}{\sigma(P D)}\right)^{2}\right]_{R_{2}} \\
& +\left[\left(\frac{R_{1, \text { model }}-R_{1, \text { max }}}{\sigma\left(R_{1}\right)}\right)^{2}+\left(\frac{R_{2, \text { model }}-R_{2, \text { max }}}{\sigma\left(R_{2}\right)}\right)^{2}\right]_{P D}
\end{aligned}
$$

To ensure that $R_{1}, R_{2}$, and $P D$ had the same weight in the cost function, the square of the residuals was normalized using the variance $\sigma^{2}$ of $R_{1}, R_{2}$, and $P D(27)$.

The entire procedure was repeated, where each of the variable parameters was varied individually, with increasingly smaller steps until the minimum residual was found. To avoid convergence to a local minimum, this procedure was repeated 100 times, after which the lowest residual was regarded as the global minimum.

The confidence interval of the optimized parameters was calculated using the finite sample confidence intervals in the maximum likelihood (25). According to this approach, the confidence region is found by varying a single parameter and minimizing all others such that the cost function remains under the value of $\chi^{2}(a, d f)$, where $a$ corresponds to the confidence level and $d f$ is the number of degrees of freedom. Using $a=0.05$ and $d f=5$, the $\chi^{2}(a, d f)$ function becomes 9.488. The Bloch simulation and minimization procedure was implemented in an in-house developed IDL program (ITT visual information solutions, Boulder, CO, USA).

\section{Calculation of Total Volumes and Regions of Interest}

Segmentation of the intracranial volume (ICV) was performed using an automatic procedure in SyMRI 7.0. The total myelin volume (MYV), cellular volume (CV), free water volume (FWV), and excess parenchymal water volume (EPWV) were calculated by summing all partial volumes within the ICV. The brain parenchymal volume (BPV) was defined as the ICV minus the total FWV. The brain parenchymal fraction (BPF) corresponds to BPV divided by ICV. The myelin fraction (MYF) was calculated as the total MYV divided by the BPV. Also, the cellular water fraction (CF) and excess parenchymal water fraction (EPWF) were calculated in a similar manner as the total $\mathrm{CV}$ divided by the BPV and total EPWV divided by the BPV, respectively.

The MWF can be derived from the model parameters because the MyW corresponds to the $\mathrm{PD}_{\mathrm{MY}}$ in the $\mathrm{V}_{\mathrm{MY}}$, and the intraand extracellular water corresponds to the sum of $\mathrm{PD}_{\mathrm{CL}}$ and $\mathrm{PD}_{\mathrm{EPW}}$ in the $\mathrm{V}_{\mathrm{CL}}$ and $\mathrm{V}_{\mathrm{EPW}}$, such that MWF for each acquisition voxel can be calculated as $\mathrm{MWF}=\left(\mathrm{V}_{\mathrm{MY}}\right.$ * $\left.\mathrm{PD} \mathrm{MY}_{\mathrm{MY}}\right) /\left(\mathrm{V}_{\mathrm{CL}}\right.$ * $\left.\mathrm{PD}_{\mathrm{CL}}+\mathrm{V}_{\mathrm{EPW}}{ }^{*} \mathrm{PD}_{\mathrm{EPW}}\right)$. Additionally, the total aqueous content of the tissue can be calculated, corresponding to the sum of the $\mathrm{MyW}$, cellular water, free water, and excess parenchymal water, $\mathrm{V}_{\mathrm{MY}}{ }^{\star} \mathrm{PD}_{\mathrm{MY}}+\mathrm{V}_{\mathrm{CL}}{ }^{*} \mathrm{PD}_{\mathrm{CL}}+\mathrm{V}_{\mathrm{FW}}{ }^{\star} \mathrm{PD}_{\mathrm{FW}}+\mathrm{V}_{\mathrm{EPW}}{ }^{\star} \mathrm{PD}_{\mathrm{EPW}}$. The total non-aqueous content then corresponds to $100 \%$ minus the aqueous content.

To define regions of interest for the spatially normalized brain images, the cropped ROI templates, based on the Wake Forrest University (WFU) PickAtlas, were taken [Ref. (21)]. To verify that the standard ROIs in spatially normalized, averaged brain images provide similar results as spatially non-normalized, separate brain images, $3 \mathrm{~mm} \times 3 \mathrm{~mm}$ ROIs were manually placed in a subset of brain structures in all participants of Ref. (21). This was also done for the three example subjects. In the MS cases, areas with MS lesions were avoided.

\section{RESULTS}

\section{Optimizing the Model Parameters to the Healthy Brain}

In Figure 3, the $\mathrm{R}_{1}, \mathrm{R}_{2}$, and $\mathrm{PD}$ values for the spatially normalized brains of the group of controls are shown as $2 \mathrm{D}$ histograms of $\mathrm{R}_{1}$ and $R_{2}, R_{1}$ and $P D$, and $R_{2}$ and $P D$. The color scale indicates the number of voxels for each coordinate in the histogram. The black dots are placed at the maximum values of the histograms in each direction, generating the 600 maxima defining the structure in the $\mathrm{R}_{1}-\mathrm{R}_{2}-\mathrm{PD}$ space.

Using these 600 maxima, the six variables in the model were optimized to find the minimum value of the cost function (See Figure 2). The values of the parameters at the minimum residual (3.446) are given in Table 1. Each parameter was varied individually while re-optimizing all others such that the cost function remained below 9.488, resulting in the determination of the SDs of the parameters, as also listed in Table $\mathbf{1}$.

\section{Behavior of the Model for the Pathological Brain}

The mean values in Table $\mathbf{1}$ provide the relaxation parameters for the four partial volumes for the healthy brain. According to the model, all observed $R_{1}, R_{2}$, and $P D$ values in the healthy brain can be reproduced by combinations of $V_{F W}, V_{C L}$, and $V_{M Y}$ using these characteristics. This is indicated as the thick black curve in Figure 4 showing the transition from $100 \% \mathrm{~V}_{\mathrm{FW}}$ at $\left(\mathrm{R}_{1}\right.$, $\left.\mathrm{R}_{2}, \mathrm{PD}\right)=\left(0.24 \mathrm{~s}^{-1}, 0.87 \mathrm{~s}^{-1}, 100 \%\right)$ to $100 \% \mathrm{~V}_{\mathrm{CL}}$ at $\left(\mathrm{R}_{1}, \mathrm{R}_{2}\right.$, $\mathrm{PD})=\left(0.78 \mathrm{~s}^{-1}, 10.3 \mathrm{~s}^{-1}, 85 \%\right)$, continuing toward $100 \% \mathrm{~V}_{\mathrm{MY}}$ at $\left(\mathrm{R}_{1}, \mathrm{R}_{2}, \mathrm{PD}\right)=\left(16.6 \mathrm{~s}^{-1}, 77 \mathrm{~s}^{-1}, 42 \%\right)$. In the Figure, the positions of $100 \% \mathrm{~V}_{\mathrm{FW}}$ and $100 \% \mathrm{~V}_{\mathrm{CL}}$ are indicated at the red dots labeled by "FW" and "CL", respectively. The $100 \% \mathrm{~V}_{\mathrm{MY}}$ position is outside the range of the plot, the grid is clipped at $40 \% \mathrm{~V}_{\mathrm{MY}}$.

For the pathological brain, two processes can occur in the model: (1) a decrease in $\mathrm{V}_{\mathrm{MY}}$ and (2) the presence of non-zero $\mathrm{V}_{\mathrm{EPW}}$. In Figure 4 a grid is displayed, indicating steps of possible combinations of $5 \%$ difference of $\mathrm{V}_{\mathrm{MY}}$ and $10 \%$ difference of $\mathrm{V}_{\mathrm{EPW}}$. This grid spans a curved surface in the $\mathrm{R}_{1}-\mathrm{R}_{2}-\mathrm{PD}$ space. In the background of Figure 4 the data for the spatially normalized brain for the MS group were plotted. It can be seen that the MS data values are shifted toward lower $\mathrm{R}_{1}$ and $\mathrm{R}_{2}$ and higher $\mathrm{PD}$ relative to the black curve, which was optimized using the healthy data values. 

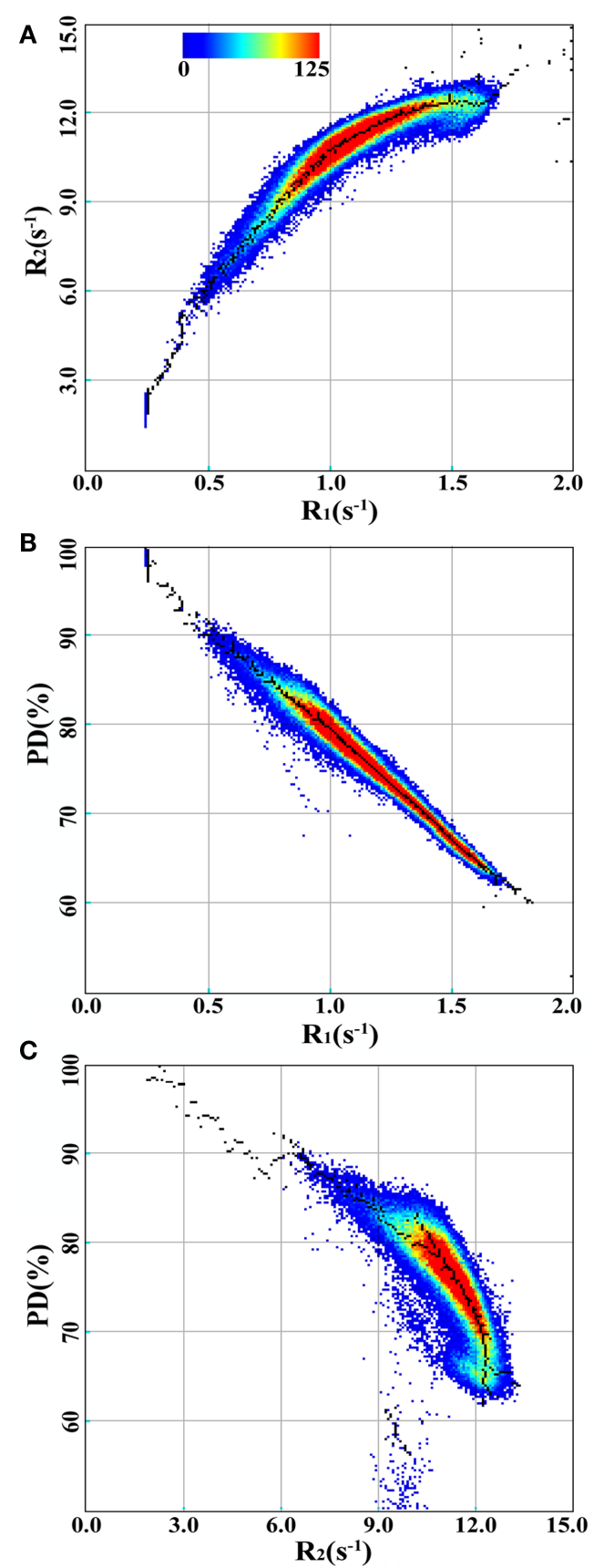

FIGURE 3 | 2D-histograms of $\mathbf{R}_{1}, \mathbf{R}_{2}$, and PD values for the spatially normalized brain images of the group of control subjects. The 2D-histograms of $R_{1}$ and $R_{2}, R_{1}$ and $P D$, and $R_{2}$ and PD are shown in (A-C), respectively. The color scale indicates the number of voxels for each coordinate. The black dots are placed at the maximum values of the 2D-histograms in each direction.

\section{Modeling the Spatially Normalized Brain Images}

The grid in Figure 4 was used to relate the $R_{1}, R_{2}$, and PD values of the spatially normalized brain data to combinations of $\mathrm{V}_{\mathrm{MY}}$, $\mathrm{V}_{\mathrm{CL}}, \mathrm{V}_{\mathrm{FW}}$, and $\mathrm{V}_{\mathrm{EPW}}$. The result is shown in Figure $\mathbf{5}$ for the spatially normalized brain images of the control and MS groups.
TABLE 1 | The parameter values of the model; on the left the fixed parameters (see MATERIALS AND METHODS); on the right, the optimized parameters where the cost function was minimized for the brain data of the control group $(n=20)$.

\begin{tabular}{|c|}
\hline Fixed parameters \\
\hline $\mathrm{R}_{2, \mathrm{MY}}=77 \mathrm{~s}^{-1}$ \\
\hline$R_{1, F W}=R_{1, E P W}=0.24 s^{-1}$ \\
\hline $\mathrm{R}_{2, \mathrm{FW}}=\mathrm{R}_{2, \mathrm{EPW}}=0.87 \mathrm{~s}^{-1}$ \\
\hline$P D_{F W}=P D_{E P W}=100 \%$ \\
\hline$k_{\mathrm{VEPW}-\mathrm{VCL}}=\infty \mathrm{s}^{-1}$ \\
\hline
\end{tabular}

Optimized parameters

$\mathrm{R}_{1, \mathrm{MY}}=16.6 \pm 13.2 \mathrm{~s}^{-1}$

$\mathrm{PD}_{\mathrm{MY}}=42 \pm 33 \%$

$k_{\mathrm{VMY}-\mathrm{VCL}}=6.7 \pm 5.2 \mathrm{~s}^{-1}$

$R_{1, C L}=0.78 \pm 0.13 s^{-1}$

$R_{2, C L}=10.3 \pm 0.6 s^{-1}$

$\mathrm{PD}_{\mathrm{CL}}=85 \pm 5 \%$

The SD of the latter values is given for a significance level of $a=0.05$.

The $\mathrm{V}_{\mathrm{MY}}$ is substantially higher for the controls than for the MS group. The total MYVs were 157 and $119 \mathrm{~mL}$, respectively, a difference of $38 \mathrm{~mL}$. Also, the total FWV was visibly lower, at $65 \mathrm{~mL}$ for the control group versus $144 \mathrm{~mL}$ for the MS group, a difference of $79 \mathrm{~mL}$. The ICV of the spatially normalized datasets was $1213 \mathrm{~mL}$ for both groups, resulting in brain volumes of 1148 and $1069 \mathrm{~mL}$, corresponding to a BPF of 94.6 and $88.1 \%$, respectively. All volumes and volume fractions in relation to brain volume are provided in Table 2.

The observed $R_{1}, R_{2}$, and $P D$ values in the standard WFU PickAtlas ROIs of separate brain structures were used to calculate the local mean $\mathrm{V}_{\mathrm{MY}}, \mathrm{V}_{\mathrm{CL}}$, and $\mathrm{V}_{\mathrm{EPW}}$ of the spatially normalized control group and spatially normalized MS group (see Table 3 ). For the healthy group, $\mathrm{V}_{\mathrm{MY}}$ for the GM structures was in the range of $8-15 \%$ (average $14 \pm 3 \%$ ), whereas that for WM structures was $18-27 \%$ (average $23 \pm 3 \%$ ). For the MS group, $\mathrm{V}_{\mathrm{MY}}$ was $1-4 \%$ lower, with most of the difference in the WM structures; the average was $13 \pm 5 \%$ for GM structures (difference: $1.6 \pm 1.5 \%$ ) and $20 \pm 3 \%$ for WM structures (difference: $2.8 \pm 1.0 \%$ ). The mean $\mathrm{V}_{\text {CL }}$ was $0-10 \%$ lower in the MS group. $\mathrm{V}_{\mathrm{EPW}}$ was higher in the MS group, with a difference of $9 \pm 10 \%$ and $5 \pm 2 \%$, respectively, compared to the healthy group. Large differences were observed for the caudate nucleus, for which the MS group had a $28 \%$ lower $\mathrm{V}_{\mathrm{CL}}$ and $31 \%$ higher $\mathrm{V}_{\mathrm{EPW}}$ compared with the healthy group. For completeness, also the MWF was derived from the model, which was $8.3 \pm 2.9 \%$ for GM structures and $14.4 \pm 2.3 \%$ for WM structures for the healthy group and $7.2 \pm 3.0 \%$ and $11.9 \pm 2.3 \%$, respectively, for the MS group, a difference of $1.2 \pm 0.9 \%$ and $2.5 \pm 0.7 \%$, respectively. The MWF values show the same trend as $\mathrm{V}_{\mathrm{MY}}$ but are substantially lower, $43 \%$ on average.

For comparison, ROIs were manually placed in a subset of all brain structures for all participants in the study, using the original, spatially non-normalized brain images (Table 4). The differences between GM and WM structures are far more extreme in this case. For example, for the healthy group, the $\mathrm{V}_{\mathrm{MY}}$ for cortical GM decreases from $15 \%$ for the standard ROI to $2 \%$ for the manually placed ROI, whereas for the corpus callosum $\mathrm{V}_{\mathrm{MY}}$ increases from 27 to $41 \%$. Most of the $V_{E P W}$ values decrease, except for the occipital WM (9\%). For the manual ROIs, no significant differences were observed for the GM structures between the MS patients and the control group. For WM, however, $\mathrm{V}_{\mathrm{MY}}$ was $3 \%$ lower for occipital WM $(p=0.04), 2 \%$ lower for frontal WM ( $p=0.04)$, and $5 \%$ lower for corpus callosum $(p=0.02)$. 

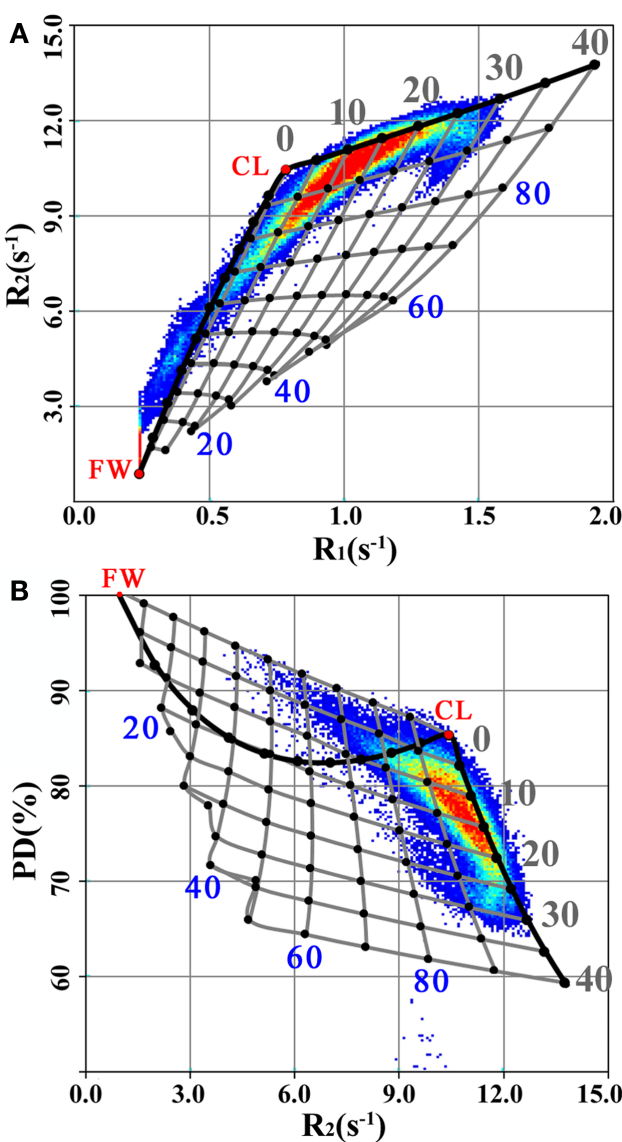

FIGURE 4 | $\mathbf{R}_{1}, \mathbf{R}_{\mathbf{2}}$, and PD values for the spatially normalized brain images of the group of MS patients, plotted in the same manner as Figure $\mathbf{3}$ for the $\mathbf{R}_{\mathbf{1}}-\mathbf{R}_{\mathbf{2}}(\mathbf{A})$ and $\mathbf{R}_{\mathbf{2}}-\mathbf{P D}(\mathrm{B})$ projections. Additionally, the thick black line indicates the transition from $100 \% V_{F W}$ (the red dot at "FW") to $100 \% V_{C L}$ (the red dot at " $C L$ ") until $40 \% V_{M Y}$, using the model parameter settings for the healthy controls (Table 1). The grid of gray lines indicates the expected changes in $R_{1}, R_{2}$, and $P D$ values for the pathological brain under myelin loss (Figure 1C) and under the presence of excess parenchymal water (Figure 1D). The cross points of the grid are placed at each $5 \%$ change in $\mathrm{V}_{\mathrm{MY}}$ and each $10 \%$ change in $\mathrm{V}_{\mathrm{EPW}}$. The $\mathrm{V}_{\mathrm{MY}}$ partial volume is indicated by the gray numbers $0-40 \%$. The $V_{\text {EPW }}$ partial volume is indicated by the blue numbers $20-80 \%$.

\section{Modeling the High-Resolution Brain Images}

In Figure 6, the model was applied on high-resolution image datasets of a middle-aged ( 45 years) and elderly control subject (72 years) and an MS patient (45 year-MS), in combination with a conventional FLAIR image (A). The $\mathrm{R}_{1}, \mathrm{R}_{2}$, and PD maps (B-D) demonstrate that the 72 year (row 2) had generally lower $R_{1}$ and $\mathrm{R}_{2}$ values and higher $\mathrm{PD}$ values throughout the brain than the 45 year (row 1 ). For the 45 year-MS (row 3 ), the $\mathrm{R}_{1}, \mathrm{R}_{2}$, and $\mathrm{PD}$ values were similar to those for the 45 year, but much lower in the areas where the MS lesions were located. Figure $6 \mathrm{E}$ presents the estimated $\mathrm{V}_{\mathrm{MY}}$, with a high $\mathrm{V}_{\mathrm{MY}}$ in the WM (33\%, see Table 5) and low $\mathrm{V}_{\mathrm{MY}}$ in the GM (4\%) for the 45 year. The 72 year showed less myelin throughout the brain than the 45 year, with an average $\mathrm{V}_{\mathrm{MY}}$ of $26 \%$ in the WM. Only the corpus callosum showed higher
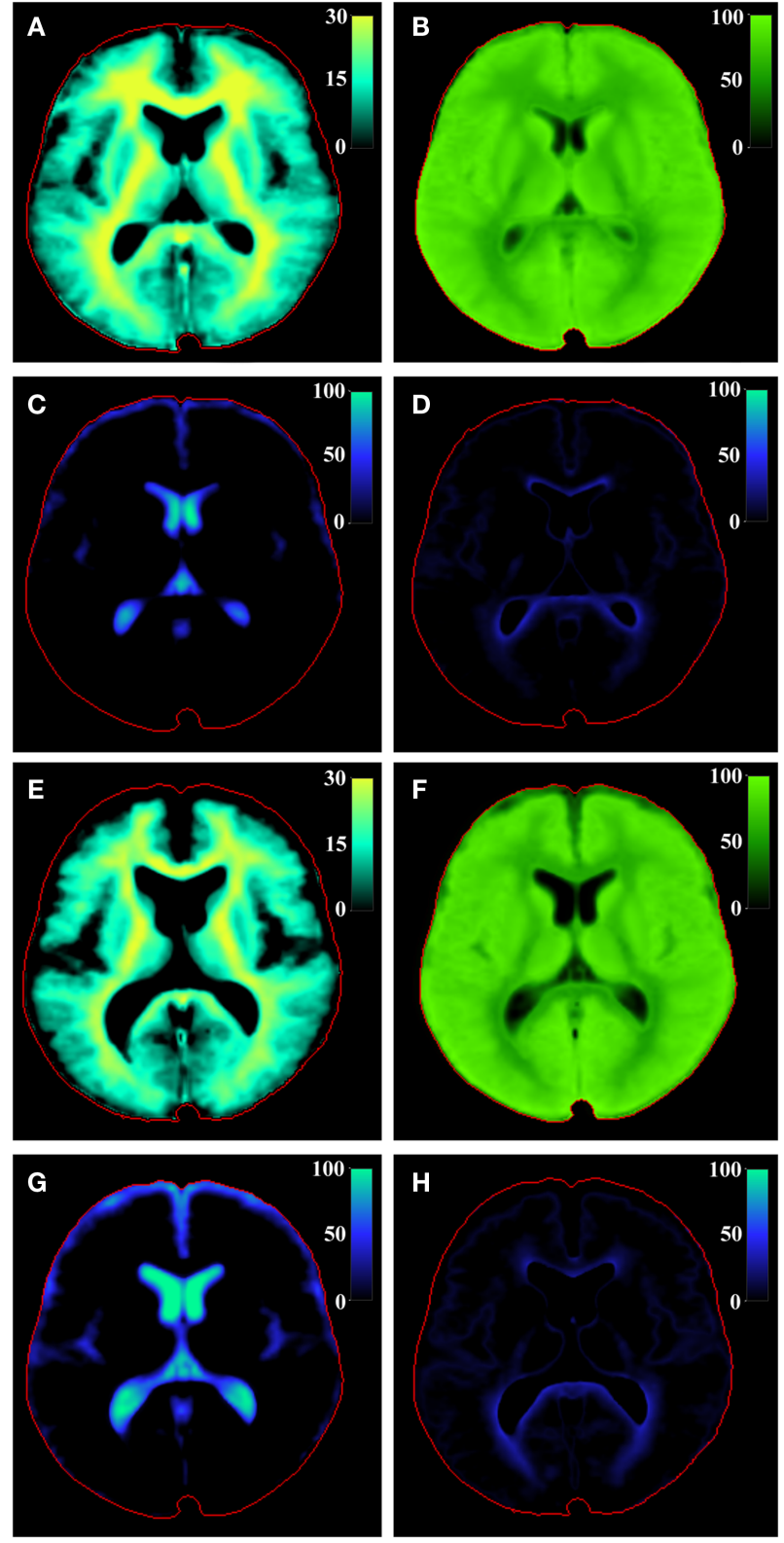

FIGURE 5 | Model calculation of (A) $V_{M Y}$, (B) $V_{C L}$, (C) $V_{F W}$, and (D) $V_{E P W}$ of the central slice of the brain of the spatially normalized group of healthy controls and of the spatially normalized group of MS patients [(E-H), respectively]. The red line indicates the intracranial volume. Note that $V_{M Y}$ is scaled to $30 \%$, whereas the other partial volumes are scaled to $100 \%$.

values (33\%). The estimated total MYVs were $155 \mathrm{~mL}$ for the 45 year, $142 \mathrm{~mL}$ for the 72 year and $119 \mathrm{~mL}$ for the 45 year-MS, corresponding to a MYF of $14.2,12.6$ and $11.5 \%$, respectively (see Table 2). The cellular fractions (Figure 6F) were 83.7, 83.7, and $84.9 \%$, respectively. Figure $6 \mathrm{G}$ presents $\mathrm{V}_{\mathrm{FW}}$, highlighting the ventricular system and periphery of the brain. Using the ICV and FWV of the subjects, the BPV can be calculated, which was $1090 \mathrm{~mL}$ for the 45 year, $1127 \mathrm{~mL}$ for the 72 year, and $1031 \mathrm{~mL}$ for the 45 year-MS. Correspondingly, the BPF was 90.3, 78.5, and $83.5 \%$, respectively. 
TABLE 2 | The total volumes and volume fractions for the spatially normalized healthy control group and spatially normalized MS group of Figures 4 and 5 as well as for the three individual subjects of Figure 6.

\begin{tabular}{|c|c|c|c|c|c|c|c|c|c|}
\hline & MYV (mL) & CV (mL) & FWV (mL) & EPWV (mL) & BPV (mL) & ICV (mL) & MYF (\%) & CF (\%) & EPWF (\%) \\
\hline Control & 157 & 934 & 65 & 57 & 1148 & 1213 & 13.7 & 81.4 & 5.0 \\
\hline MS & 119 & 872 & 144 & 78 & 1069 & 1213 & 11.1 & 81.6 & 7.3 \\
\hline 45 year & 155 & 911 & 117 & 24 & 1090 & 1207 & 14.2 & 83.6 & 2.2 \\
\hline 72 year & 142 & 944 & 308 & 41 & 1127 & 1435 & 12.6 & 83.7 & 3.7 \\
\hline 45 year-MS & 119 & 875 & 204 & 37 & 1031 & 1234 & 11.5 & 84.9 & 3.6 \\
\hline
\end{tabular}

Listed are the total myelin volume (MY), cellular volume (CV), free water volume (FWV), excess parenchymal water volume (EPWV), total brain volume (BPV), and intracranial volume (ICV). The volume components that constitute the brain were normalized on BPV, resulting in the myelin fraction (MYF), cellular fraction (CF), and excess parenchymal water fraction (EPWF) of the brain.

TABLE 3 | The mean myelin partial volume $\mathbf{V}_{\mathrm{MY}}$, cellular partial volume $\mathbf{V}_{\mathrm{CL}}$, and the excess parenchymal water partial volume $\mathbf{V}_{\mathrm{EPW}}$ of various brain structures, estimated as a percentage of the acquisition voxel volume.

\begin{tabular}{|c|c|c|c|c|c|c|c|c|}
\hline & \multicolumn{4}{|c|}{ Healthy controls } & \multicolumn{4}{|c|}{ Multiple sclerosis patients } \\
\hline & $\mathrm{V}_{\mathrm{MY}}(\%)$ & $\mathrm{V}_{\mathrm{CL}}(\%)$ & $\mathrm{V}_{\mathrm{EPW}}(\%)$ & MWF (\%) & $\mathrm{V}_{\mathrm{MY}}(\%)$ & $\mathrm{V}_{\mathrm{CL}}(\%)$ & $\mathbf{V}_{\text {EPW }}(\%)$ & MWF (\%) \\
\hline Insula & 8 & 75 & 17 & 4 & 8 & 66 & 26 & 4 \\
\hline Cingulate cortex & 12 & 81 & 7 & 7 & 8 & 78 & 14 & 4 \\
\hline Caudate nucleus & 9 & 87 & 4 & 5 & 6 & 59 & 35 & 3 \\
\hline Cortical gray matter & 15 & 74 & 11 & 9 & 14 & 66 & 20 & 8 \\
\hline Pons & 18 & 69 & 13 & 11 & 17 & 60 & 23 & 10 \\
\hline Putamen & 15 & 85 & 0 & 9 & 15 & 85 & 0 & 9 \\
\hline Mid brain & 19 & 81 & 0 & 12 & 18 & 79 & 3 & 11 \\
\hline Thalamus & 19 & 81 & 0 & 12 & 16 & 84 & 0 & 9 \\
\hline Occipital white matter & 18 & 82 & 0 & 11 & 15 & 83 & 2 & 9 \\
\hline Frontal white matter & 21 & 77 & 2 & 14 & 19 & 73 & 8 & 11 \\
\hline Parietal white matter & 21 & 77 & 2 & 14 & 19 & 73 & 8 & 11 \\
\hline Sub-lobar white matter & 25 & 66 & 9 & 16 & 21 & 65 & 14 & 13 \\
\hline White matter & 23 & 75 & 2 & 15 & 19 & 73 & 8 & 11 \\
\hline Corpus callosum & 27 & 60 & 13 & 18 & 25 & 55 & 20 & 16 \\
\hline
\end{tabular}

The values were calculated using the proposed model and the reported relaxation rates $R_{1}$ and $R_{2}$ and proton density PD in the WFU Pickatlas ROIs of the spatially normalized, averaged group of healthy controls and the spatially normalized, averaged group of multiple sclerosis patients from Ref. (21) (Table 2, cropped ROI templates). Added are the expected myelin water fraction MWF values, calculated as $P D_{M Y} /\left(P D_{C L}+P D_{E P W}\right)$.

TABLE 4 | The mean myelin partial volume $V_{M Y}$, cellular partial volume $V_{C L}$, and the excess parenchymal water partial volume $V_{E P W}$ of various brain structures, estimated as a percentage of the acquisition voxel volume.

\begin{tabular}{|c|c|c|c|c|c|c|c|c|}
\hline & \multicolumn{4}{|c|}{ Healthy controls } & \multicolumn{4}{|c|}{ Multiple sclerosis patients } \\
\hline & $\mathrm{V}_{\mathrm{MY}}(\%)$ & $\mathrm{V}_{\mathrm{CL}}(\%)$ & $\mathrm{V}_{\text {EPW }}(\%)$ & MWF (\%) & $\mathrm{V}_{\mathrm{MY}}(\%)$ & $\mathrm{V}_{\mathrm{CL}}(\%)$ & $\mathrm{V}_{\mathrm{EPW}}(\%)$ & MWF (\%) \\
\hline Cingulate cortex & 2 & 96 & 2 & 1 & 2 & 95 & 3 & 1 \\
\hline Caudate nucleus & 8 & 92 & 0 & 4 & 9 & 91 & 0 & 5 \\
\hline Cortical gray matter & 2 & 95 & 3 & 1 & 2 & 95 & 3 & 1 \\
\hline Putamen & 11 & 89 & 0 & 6 & 10 & 90 & 0 & 5 \\
\hline Thalamus & 19 & 81 & 0 & 12 & 15 & 84 & 1 & 9 \\
\hline Occipital white matter & 34 & 57 & 9 & 25 & 31 & 61 & 8 & 22 \\
\hline Frontal white matter & 36 & 62 & 2 & 28 & 34 & 64 & 2 & 25 \\
\hline Corpus callosum & 41 & 56 & 3 & 35 & 36 & 60 & 4 & 29 \\
\hline
\end{tabular}

The values were calculated using the proposed model and the relaxation rates $R_{1}$ and $R_{2}$ and proton density PD in manually placed $R O / s$ in all participants of Ref. (21). Added are the expected myelin water fraction MWF values, calculated as $P D_{M Y} /\left(P D_{C L}+P D_{E P W}\right)$.

The 45 year exhibited a small amount of $\mathrm{V}_{\mathrm{EPW}}$ (Figure $\mathbf{6 H}$ ), mainly around the occipital horns of the lateral ventricles, with a maximum of $11 \%$ in the occipital WM. The 72 year had elevated $\mathrm{V}_{\mathrm{EPW}}$ in the complete periventricular region, with values of up to $16 \%$ partial volume. The 45 year-MS showed moderate $V_{\text {EPW }}$ values at the periventricular area and $12 \%$ in the occipital WM. At the location of MS lesions, however, high $\mathrm{V}_{\mathrm{EPW}}$ values, up to approximately $50 \%$ were observed. The $\mathrm{V}_{\mathrm{EPW}}$ volumes were $24 \mathrm{~mL}$ for the $45 \mathrm{y}, 41 \mathrm{~mL}$ for the $72 \mathrm{y}$, and $37 \mathrm{~mL}$ for the 45 year-MS, corresponding to an EPWF of 2.2, 3.5, and 3.6\%, respectively.

The histograms of $\mathrm{V}_{\mathrm{MY}}, \mathrm{V}_{\mathrm{CL}}, \mathrm{V}_{\mathrm{FW}}$, and $\mathrm{V}_{\mathrm{EPW}}$ are shown in Figure 7 to assess the distribution of the partial volumes of the three subjects. The histograms contain 100 bins from 0 to $100 \%$ partial volume and are plotted as a percentage of the ICV volume to compensate for the difference in subject head size. The 45 year exhibited two peaks in the $\mathrm{V}_{\mathrm{MY}}$ histogram at 5 and $32 \% \mathrm{~V}_{\mathrm{MY}}$. For 


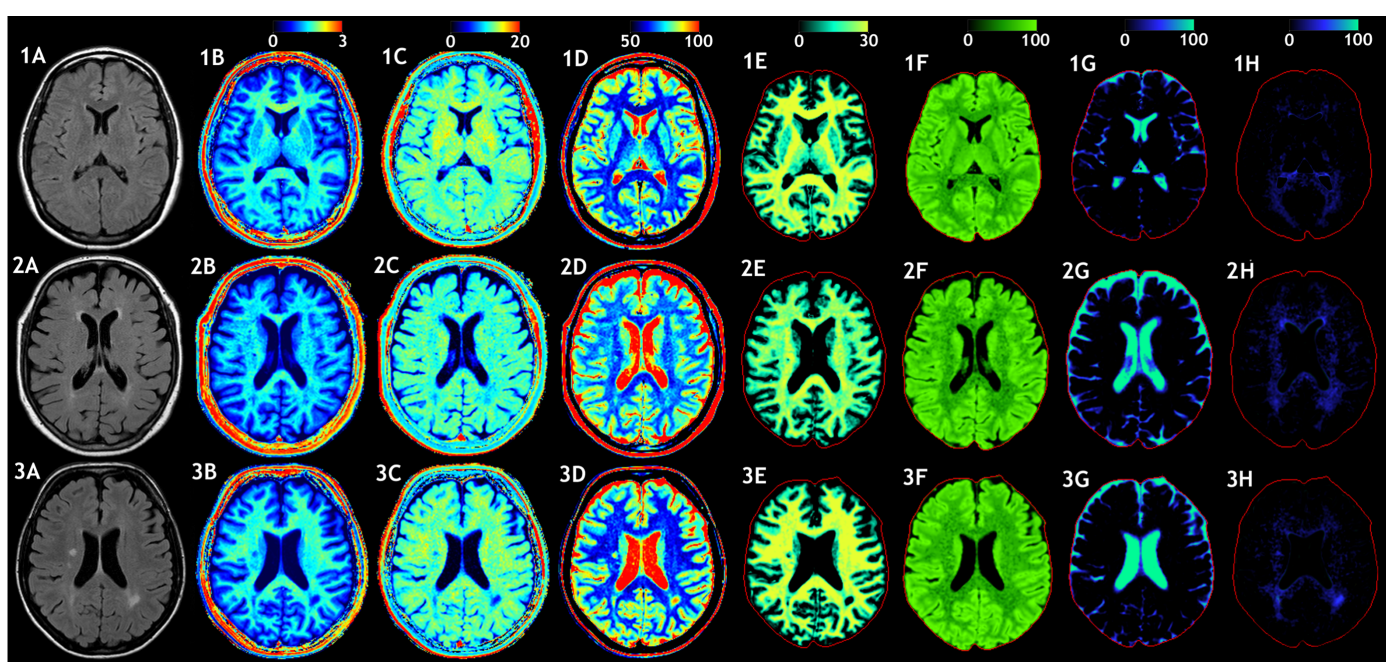

FIGURE 6 | Examples of the model calculation on an axial slice of the brain. (row 1) Healthy subject, female 45 years old, (row 2) elderly control subject, female 72 years old, and (row 3) patient, female, 45 years old, diagnosed with secondary progressive MS. (A) A conventional FLAIR image of the same slice is added as a visual reference. (B) The measured $R_{1}$ relaxation rate is shown on a scale of $0-3 s^{-1}$, (C) the $R_{2}$ relaxation rate is shown on a scale of $0-20 s^{-1}$, and (D) the proton density PD is shown on a scale of $50-100 \%$, where $100 \%$ corresponds to pure water at $37^{\circ} \mathrm{C}$. (E) Using the $R_{1}, R_{2}$, and PD values in combination with the look-up grid of Figure $\mathbf{4}$ the myelin partial volume $V_{M Y}$ was calculated, as shown on a scale of 0-30\%, (F) the cellular partial volume $V_{C L}$, (G) free water partial volume $V_{F W}$, and $\mathbf{( H )}$ excess parenchymal water partial volume $V_{E P W}$ were all calculated all on a scale $0-100 \%$. The red intracranial cavity outline is displayed in all tissue images for visual guidance.

TABLE 5 | The mean myelin partial volume $V_{M Y}$, cellular partial volume $V_{C L}$, the excess parenchymal water partial volume $V_{E P W}$, and myelin water fraction MWF of various brain structures, estimated as a percentage of the acquisition voxel volume for the three example subjects.

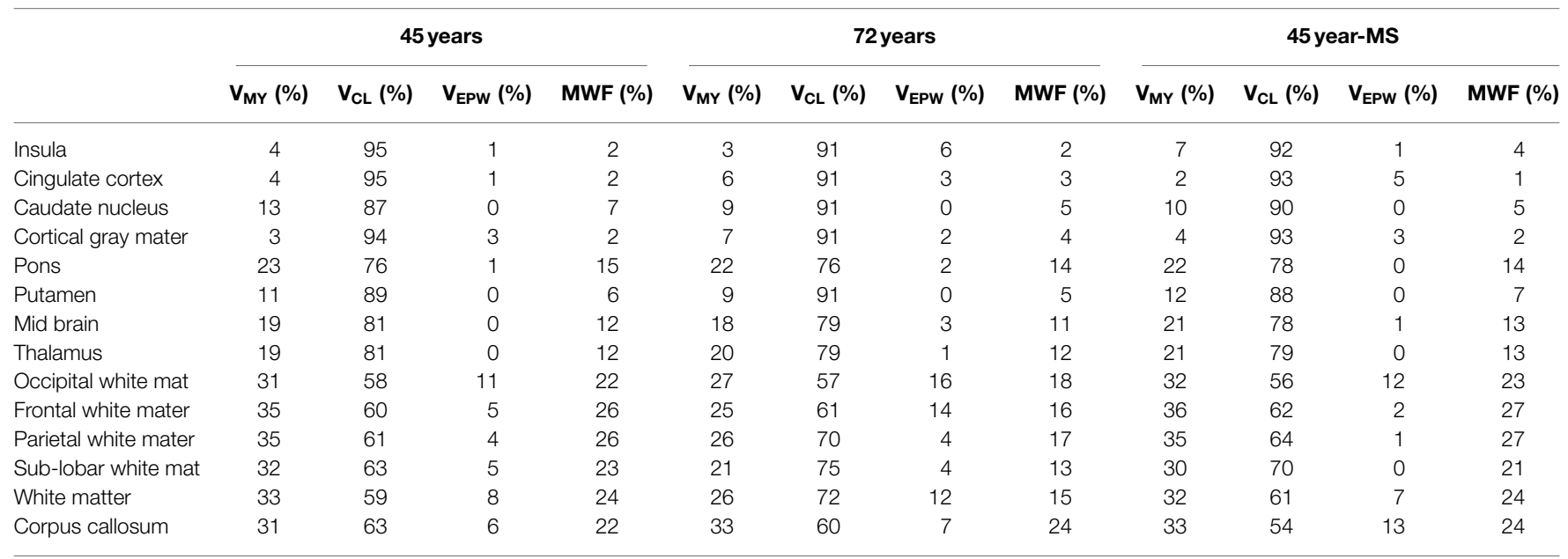

the 72 year, the peak $\mathrm{V}_{\mathrm{MY}}$ values occurred at $25 \%$. The 45 yearMS did not have a clear peak at higher $\mathrm{V}_{\mathrm{MY}}$ values. The $\mathrm{V}_{\mathrm{CL}}$ values peaked at 68 and $92 \%$ for the 45 year, but only one peak was observed for both the 72 and 45 year-MS at $89 \%$. $V_{\mathrm{FW}}$ values were generally low $(<0.5 \%)$ in the complete range but exhibited a sharp peak at $100 \% \mathrm{~V}_{\mathrm{FW}}$, with a maximum of $3.7 \%$ for the 45 year, $23.3 \%$ for the 72 year, and $11.9 \%$ for the 45 year-MS. $V_{\text {EPW }}$ was observed in all three subjects, but the values were lowest for the 45 year.

The area with the lesion of the MS patient, posterior to the left lateral ventricle, was zoomed out and displayed in Figure 8, showing a FLAIR image together with $\mathrm{V}_{\mathrm{MY}}, \mathrm{V}_{\mathrm{CL}}, \mathrm{V}_{\mathrm{FW}}$, and $\mathrm{V}_{\mathrm{EPW}}$, taken from Figures 6A,E-H. At the location of the FLAIR hyperintensity, the $V_{M Y}$ was equal to 0 , whereas the $V_{E P W}$ values were up to $55 \%$ partial volume. The diffuse hyper-intensity, located between the lesion and lateral ventricle, exhibited $V_{M Y}$ values of $15-20 \%$ and $V_{\text {EPW }}$ values of $25-30 \%$ partial volume. Elevated $\mathrm{V}_{\mathrm{EPW}}$ values were observed in a large area around the lesion. The $\mathrm{V}_{\mathrm{CL}}$ varied only slightly, ranging between $45 \%$ at the lesion and $55 \%$ at the diffusely hyper-intense area.

Using the four partial volumes, the total aqueous content of the brain can be derived. The sum of all PD contributions of $\mathrm{V}_{\mathrm{MY}}, \mathrm{V}_{\mathrm{CL}}$, $\mathrm{V}_{\mathrm{FW}}$, and $\mathrm{V}_{\mathrm{EPW}}$ is shown in Figure 9A for the 45 year-MS, for the same slice as Figures 6 and $\mathbf{8}$. The centers of the MS lesions exhibit a total aqueous content of 85-95\%, consisting entirely of the PD component of $\mathrm{V}_{\mathrm{CL}}$ and $\mathrm{V}_{\mathrm{EPW}}$. Normal appearing $\mathrm{WM}$ for this patient showed not only a total aqueous content approximately 

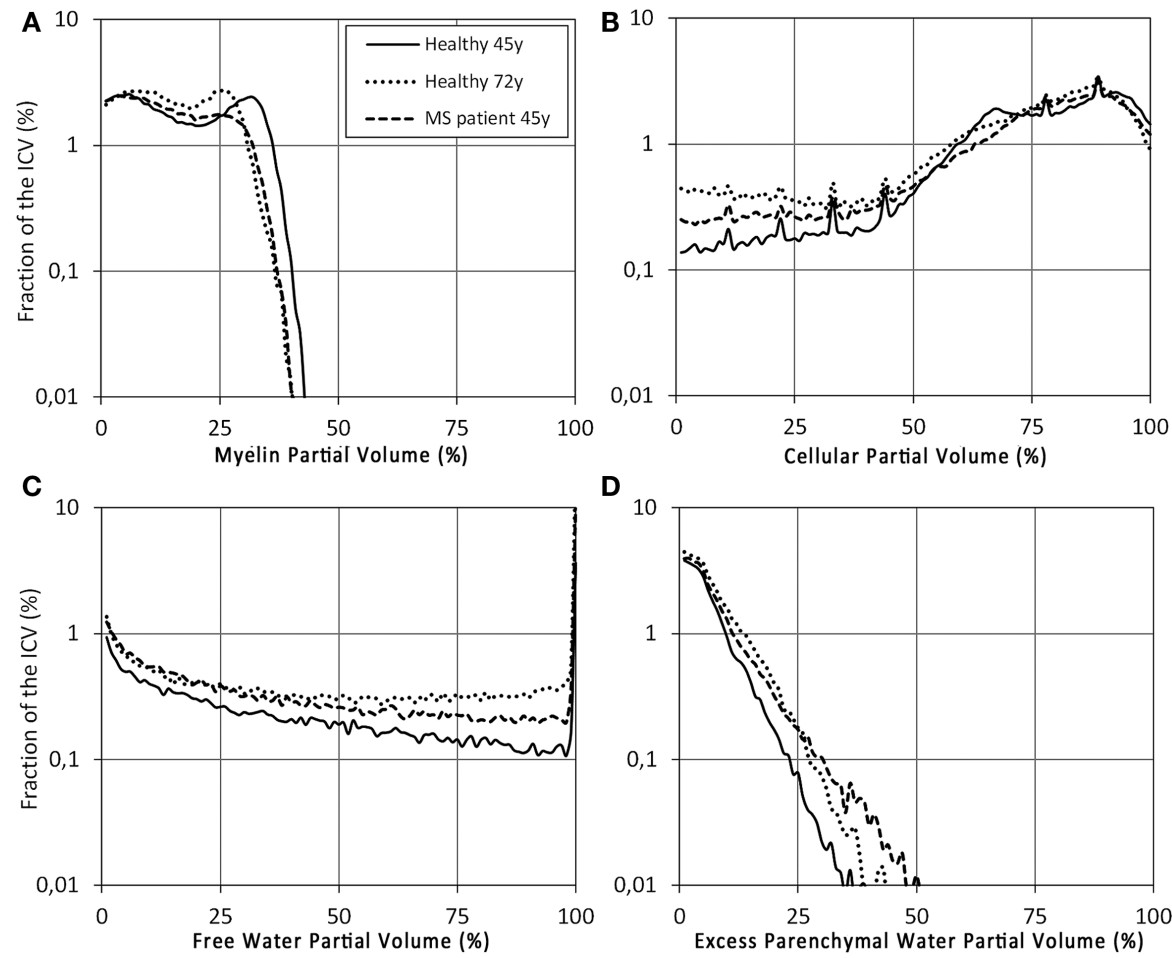

FIGURE 7 | Histograms of the (A) $\mathbf{V}_{\mathrm{MY}}$, (B) $\mathbf{V}_{\mathrm{CL}}$, (C) $\mathbf{V}_{\mathrm{FW}}$, and (D) $\mathbf{V}_{\mathrm{EPW}}$ partial volume distributions of the control subject (solid line), elderly control subject (dotted line), and MS patient (dashed line) from Figure 6. The $x$-axis was divided into 100 bins of $1 \%$ partial volume over the range $0-100 \%$. The scaling on the $y$-axis is logarithmic, as a percentage of the ICV.

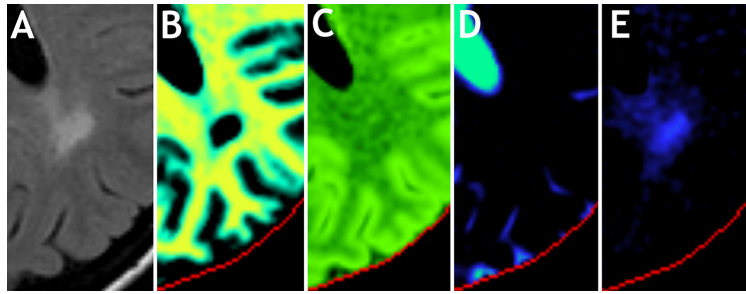

FIGURE 8 | Zoomed part on an MS lesion of the patient in Figure 6, row 3. Shown are (A) the conventional FLAIR image, (B) myelin partial volume $V_{M Y}$, (C) cellular partial volume $V_{C L}$, (D) free water partial volume $V_{F W}$, and $(\mathbf{E})$ excess parenchymal water partial volume $V_{\text {EPW. }}$. Color scaling is identical to Figure 6.

$70 \%$, consisting mainly of the $\mathrm{PD}$ component of $\mathrm{V}_{\mathrm{MY}}$ and $\mathrm{V}_{\mathrm{CL}}$ but also a minor contribution of $\mathrm{V}_{\mathrm{EPW}}$ in the order of $5 \%$. Normal appearing GM shows a total aqueous content of approximately $85 \%$, consisting largely of the $\mathrm{PD}$ component of $\mathrm{V}_{\mathrm{CL}}$, but with a small contribution of $\mathrm{V}_{\mathrm{MY}}$, up to $5 \%$. The remaining non-aqueous content is shown in Figure 9B.

\section{DISCUSSION}

In the present study, the $R_{1}, R_{2}$, and PD values, as measured in the brain using a fast multi-parametric qMRI sequence, were modeled by four partial volume compartments per acquisition voxel, (1) the myelin partial volume $\mathrm{V}_{\mathrm{MY}}$, (2) cellular partial volume $\mathrm{V}_{\mathrm{CL}}$, (3) free water partial volume $\mathrm{V}_{\mathrm{FW}}$, and (4) excess parenchymal water partial volume $\mathrm{V}_{\mathrm{EPW}}$. The major advantage of this model is that it produces an estimate of three clinically important parameters, the total brain volume, the degree of myelination of the brain parenchyma, and the degree of edema of the brain parenchyma, based on a single, relatively short acquisition.

For a complex organ, such as the brain, with an abundance of magnetically interacting microscopic substructures, MR signal relaxation will behave as a multitude of exponentials. Multicomponent measurements, such as the multi-exponential $\mathrm{T}_{2}$ relaxation and mcDESPOT approaches, typically regularize relaxation signals to force the solution into a fast component attributed to MyW, a medium-time component attributed to intra- and extracellular water and occasionally in a long-time component attributed to CSF. Attempts to experimentally resolve the fast component, however, are very challenging. The qMRI sequence employed in this work cannot resolve the fast signal component, but can accurately measure the medium-time relaxation component (28). The estimation of myelin partial volume of our model is, therefore, based on the shift of this medium-time component due to magnetization exchange between MyW and surrounding intra- and extracellular water. Such a shift is observable both in the $R_{1}$ and $R_{2}$ relaxation rates, thus, building a specific pattern in the $R_{1}-R_{2}-P D$ space, as visualized in Figure 3 for a group of healthy controls and in Figure 4 for a group of MS patients. Therefore, the model relies on a combined $\mathrm{R}_{1}-\mathrm{R}_{2}-\mathrm{PD}$ measurement as a single component/multi-parametric quantification strategy, in contrast to the multi-component/single parametric quantification methods, such as the multi-component $\mathrm{T}_{2}$ relaxation. The 

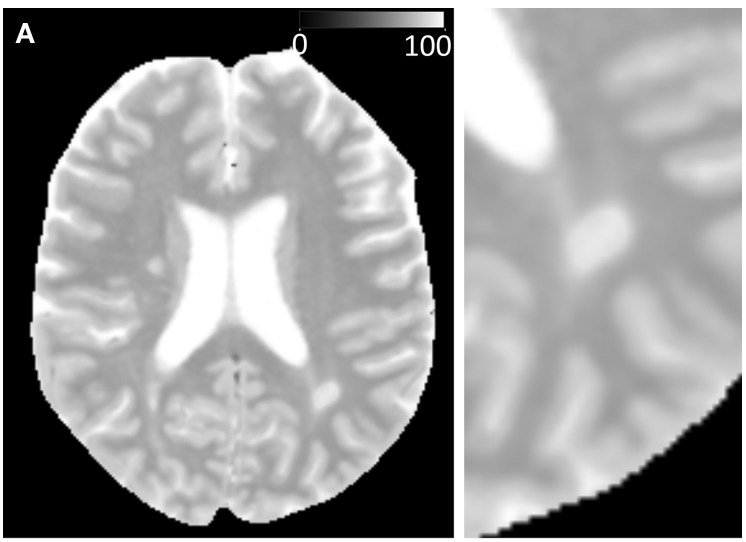

B 0 100

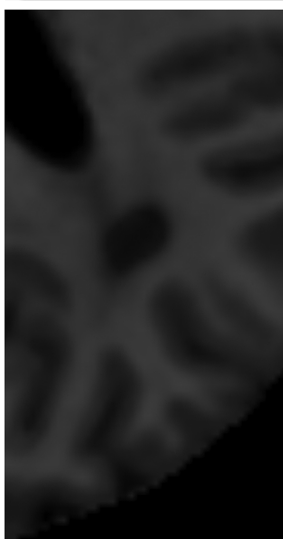

FIGURE 9 | Calculated total aqueous content (A), corresponding to the sum of myelin water, cellular water, free water and excess parenchymal water, and the remaining, total non-aqueous content (B) of the $\mathbf{4 5}$ year-MS patient. The same slice and zoomed part are displayed as in Figures $\mathbf{6}$ and $\mathbf{8}$

observed values for brain parenchyma of $\mathrm{R}_{1}$ in the range of $0.9-1.9 \mathrm{~s}^{-1}\left(\mathrm{~T}_{1}=530-1100 \mathrm{~ms}\right)$ and $\mathrm{R}_{2}$ in the range of $10.5-13 \mathrm{~s}^{-1}$ $\left(\mathrm{T}_{2}=75-95 \mathrm{~ms}\right)$ corresponded well with previously reported values for GM and WM $(29,30)$, where other qMRI methods were used. Also, the measured PD corresponds well to the reported values with GM structures of $80-86 \%$ and WM of $74-76 \%(31,32)$.

The determined optimal parameter values for the partial volume compartments are listed in Table 1. The result of the optimization provides three specific coordinates in the $R_{1}-R_{2}-P D$ space, for pure $V_{F W}$ [set by literature values to $\left(R_{1}\right.$, $\left.\mathrm{R}_{2}, \mathrm{PD}\right)=\left(0.24 \mathrm{~s}^{-1}, 0.87 \mathrm{~s}^{-1}, 100 \%\right)$ ], pure $\mathrm{V}_{\mathrm{CL}}$ [estimated at $\left(0.78 \mathrm{~s}^{-1}, 10.3 \mathrm{~s}^{-1}, 85 \%\right)$ ] and pure $\mathrm{V}_{\mathrm{MY}}$ [estimated at $\left(16.6 \mathrm{~s}^{-1}\right.$, $\left.\left.77 \mathrm{~s}^{-1}, 42 \%\right)\right]$. The characteristics of the $\mathrm{V}_{\mathrm{CL}}$ are close to those of cortical GM $(20,29,30)$. The characteristics of the $V_{M Y}$ are within the range of previous reported values $(11,22)$. Using the model, the possible value combinations of $\mathrm{R}_{1}, \mathrm{R}_{2}$, and $\mathrm{PD}$ in the healthy brain were visualized by the solid black curve through the $\mathrm{R}_{1}-\mathrm{R}_{2}-\mathrm{PD}$ space, as plotted in Figure 4 . The difference between the healthy brain and pathological brain was described using two components: (1) the variation of the $\mathrm{V}_{\mathrm{MY}}$, indicating myelin loss, and (2) the presence of $\mathrm{V}_{\mathrm{EPW}}$, indicating the presence of edema. These two components expanded the (healthy) curve to a curved surface grid, as shown in Figure 4. Each observed value combination of $R_{1}, R_{2}$, and $P D$ in acquisition voxels of a pathological brain is regarded as a combination of the $\mathrm{V}_{\mathrm{MY}}$, $\mathrm{V}_{\mathrm{CL}}, \mathrm{V}_{\mathrm{FW}}$, and $\mathrm{V}_{\mathrm{EPW}}$ partial volume compartments. As shown in Figure 5, substantial differences were observed between the spatially normalized control group and spatially normalized MS group in all partial volumes. The MS group had a smaller $\mathrm{V}_{\mathrm{MY}}$ and $\mathrm{V}_{C L}$ (a difference of 3.1 and $5.1 \%$ of the ICV, respectively) and larger $\mathrm{V}_{\mathrm{FW}}$ and $\mathrm{V}_{\mathrm{EPW}}$ (a difference of 6.5 and $1.7 \%$ of the ICV, respectively). Consequently, the average brain volume of the MS group was smaller than that of the control group $(88.1 \%$ versus $94.6 \%$ of the ICV), the degree of myelination in the brain was lower (11.1\% versus $13.7 \%$ of the BPV) and the degree of edema in the brain was higher ( $7.3 \%$ versus $5.0 \%$ of the BPV). This result is congruent with knowledge concerning the disease progression of MS (3-5). The relative CV in the brain was virtually identical ( 81.6 and $81.4 \%$ ), as can be expected in a model where edema is described by a separate class of excess parenchymal water, which is an addition of water to the normal cellular partial volume. The values in Table 3 for the various brain structures confirm the image shown in Figure 5.

The model was tested on three individual subjects as examples for high-resolution imaging. This can by no means be representative for entire groups of subjects and, hence, is purely used as example of the application of the model. Inclusion of larger groups to assess statistical differences with different age groups and diseases will be performed in future work. Clear differences were observed among the three subjects. Compared with the healthy controls, the $\mathrm{V}_{\mathrm{MY}}$ partial volume was lower for both the elderly subject and MS patient (Figure 6). Additionally, the MS patient showed strong local decreases at the location of MS lesions. Similar to the spatially normalized brains of Figure 5, the cellular fraction of the brain was virtually identical for all subjects. The $\mathrm{V}_{\mathrm{FW}}$ clearly highlights the CSF in the ventricular system and brain periphery, making it possible to calculate the brain volume of the subjects. The elderly subject had the smallest brain, with a BPF of $78.5 \%$, compared with the $90.3 \%$ for the healthy 45 year and $83.5 \%$ for the MS patient. Simultaneously, the MS patient had the lowest myelination, with a MYF of $11.5 \%$, compared with $14.2 \%$ for the healthy 45 year and $12.6 \%$ for the 72 year. In Figure 7 the cause of the reduction can be attributed to a substantial loss of high $\mathrm{V}_{\mathrm{MY}}$ values for both the MS patient and 72 year. The EPWF was substantially higher for the 72 year and the 45 year-MS compared with the healthy 45 year. These findings are consistent with general myelin loss and edema during aging and MS disease progression.

The behavior of the partial volume components around the MS lesion of the 45 year-MS, displayed in the zoomed sections shown in Figure 8, is particularly interesting. The hyper-intensity on the FLAIR image has diffuse edges, making it difficult to estimate the exact volume of the lesion. However, on the $\mathrm{V}_{\mathrm{MY}}$ image, a clear center, where the myelin has completely vanished, can be observed. At the same location, there is an elevation of the $V_{\mathrm{EPW}}$, but this area is larger and decreases toward 0 outwards. On a FLAIR image, no distinction can be made between edema and myelin loss because both processes result in a hyper-intense signal. Using the model, on the other hand, the partial volume images 
indicate a demyelinated center within a larger area of edema. This example suggests that the model can distinguish between myelin loss and the presence of excess water in edema.

An interesting derivate of the model is the total aqueous content and the corresponding, remaining non-aqueous content. The used sequence cannot resolve the short $\mathrm{R}_{2}$ relaxation component and, therefore, the observed PD value will correspond to the visible PD of the medium and long-time components. Using the observed shift in $\mathrm{R}_{1}$ and $\mathrm{R}_{2}$ the model can predict the presence of the myelin component and, therefore, the true PD value as would be measured at an echo time of 0 . The non-aqueous content (Figure 9B) can be attributed to the presence of macromolecules in the brain. From the results, it can be derived that the macromolecular content for the 45 year-MS in the MS lesions was $15-5 \%$, of normal appearing WM approximately $30 \%$, and of normal appearing GM approximately $15 \%$. These results are very similar to the reported values of Mezer et al. (33) and Abbas et al. (34). Our intention is to validate our results further on larger groups of MS patients in future work. Within the possible restrictions of ethical permission, the actual myelin content must be validated by histopathology in combination with the selective staining of individual tissue components.

In Table 2 the MWF is also listed, as directly derived from the model PD values. The definitions of $\mathrm{V}_{\mathrm{MY}}$ and MWF are not identical; $\mathrm{V}_{\mathrm{MY}}$ is the estimated MYF of an acquisition voxel based on the effective relaxation properties of that voxel, whereas MWF corresponds to the ratio of observable short-time relaxation (myelin) and medium-time relaxation (cellular) water content. The calculated MWF values are considerably lower than $\mathrm{V}_{\mathrm{MY}}(43 \%$ on average). The cause is that MyW only covers a fraction of the total MYV, which also includes the (nonobservable) myelin semi-solids. An issue reported by Zhang et al. (35), however, may cause a difference between our observed MWF and the reported MWF values: Using the multi-echo $\mathrm{T}_{2}$ relaxation in combination with the NNLS method, the magnetization exchange, responsible for the shift of the medium-time component, is ignored. Such an exchange not only results in a shift of the medium-time component, but is also responsible for a simultaneous decrease in the short-time component. This will lead to a lower observed value for MWF. Studies measuring MWF using multi-exponential $\mathrm{T}_{2}$ relaxation indeed reported lower values than our estimated MWF values, such as $7.0-10.1 \%$ in $\mathrm{WM}, 3.6-5.6 \%$ in the putamen, and $4.5-4.7 \%$ in the thalamus $(8,10,36-38)$, compared with our values of 15,9 , and $12 \%$, respectively (Table 3 ). By contrast, the mcDESPOT approach does account for magnetization exchange and consequently exhibits considerably higher values of MWF. For example, the observed MWF values were as high as $28-30 \%$ for WM, $11-13 \%$ for the putamen, and $14-15 \%$ for the thalamus (13), which are more in the range of our estimated $V_{M Y}$ values. In our opinion, this discrepancy is a highly interesting field that must be explored and further understood. A thorough validation study on patients and healthy controls using our method will be the subject of future research.

A limitation of our approach is that the model had to be grossly simplified in order to provide any reasonable results. Each compartment can have very different behavior throughout the brain and with various diseases. Magnetic interaction was reduced to two exchange rates and a number of parameters were fixed to reasonable, but unvalidated values. Adding more degrees of freedom, however, would make it impossible for the model to converge to a solution. The used spatial normalization process resulted in a low resolution of the brain images. This inevitably led to the loss of anatomical detail and smearing of tissue characteristics, which can explain the differences between the values of Table 3 and Tables 4 and 5. For example, voxels that are partially filled with bulk CSF at the periphery of the brain may be seen at low resolution as brain tissue with $\mathrm{V}_{\mathrm{EPW}}$. Indeed, the spatially normalized brains of the controls in Figure $\mathbf{5}$ had a relatively high amount of $\mathrm{V}_{\mathrm{EPW}}$ at $57 \mathrm{~mL}$, whereas all three individual examples in Figure $\mathbf{6}$ had much lower values. The estimated $V_{\text {EPW }}$ for healthy controls in Table 2 was high for the insula, cortical GM, pons, and corpus callosum; the caudate nucleus showed an extreme value for the MS group. All of these structures interface with bulk CSF and, hence, the $V_{\text {EPW }}$ likely is lower in reality. Also, GM and WM structures may be blended at this resolution. For the spatially normalized brain images of the healthy controls cortical GM had 15\% and WM had 23\% $\mathrm{V}_{\mathrm{MY}}$, whereas the differences between GM and WM for all subjects in Ref. (21), and for the three example subjects, were much more extreme, $2-7 \%$ for GM and $26-41 \%$ for WM. In Figure 7, $\mathrm{V}_{\mathrm{MY}}$ peaks can be observed at 5 and $32 \%$ for the 45 year (6A), and $V_{C L}$ peaks can be observed at 68 and $92 \%(6 \mathrm{~B})$, which are likely to be centered at GM and WM. A higher resolution of the spatial normalization procedure would likely change the values of Table 3 and make them more similar to those of Tables 4 and 5. Future work will focus on high-resolution spatial normalization in combination with a better definition for the regions of interest to improve the distinction between the various brain structures. Standard, template ROIs have an advantage over (timeconsuming and user-dependent) manually placed ROIs, but our data show that the loss of anatomic detail has a large effect on the results.

Another limitation of our method is that the measured $V_{M Y}$ properties in Table 1 have large SDs. This is a result of the relatively shallow minimum in the optimization, where a change in one parameter can be compensated for by a change in other parameters. Therefore, our model cannot accurately determine the characteristics of pure $V_{M Y}$. The effect of parameter changes in $V_{M Y}$ on the calculated grid in Figure 4, however, is relatively small. Brain parenchyma typically has $<30 \% \mathrm{~V}_{\mathrm{MY}}$, and substantial changes near $100 \% \mathrm{~V}_{\mathrm{MY}}$ only have a small effect at lower values. For example, when perturbing $\mathrm{R}_{1, \mathrm{MY}}$ and $\mathrm{PD}_{\mathrm{MY}}$ by one $\mathrm{SD}$, the grid points of $\mathrm{V}_{\mathrm{MY}}$ in Figure 4 changed by $<5 \%$, indicating that our model is relatively robust for practical purposes.

All parameters of the model were adapted to $1.5 \mathrm{~T}$ spatially normalized data. Because relaxation rates change with field strength, the modeled grid from Figure 4 must be re-optimized for other field strengths. Furthermore, it is important to realize that the partial volumes are measured by observation of magnetic properties of the brain. The fast-relaxing, non-observable MyW has a magnetization exchange with the surrounding cellular water, resulting in an increase in the effective relaxation rate of cellular water in the vicinity. This effect will decrease with distance and, 
thus, cannot define a hard boundary. Therefore, myelin partial volume in the acquisition voxel reflects the extent of the effect of magnetization exchange in space rather than defining a physical boundary of the myelin sheets. Using this argument, it is not likely that the measured total MYV using this model is identical to the total MYV, which could be measured by summing the volumes of all myelin sheets under a microscope.

For quantitative monitoring of patients in clinical routine, we consider it important that not only the brain volume is monitored. Although this is an important clinical measure, it is only a volumetric measure. It does not reveal any pathological changes in the tissue composition of the brain. Neurological degeneration is related to differences in $R_{1}, R_{2}$, and $P D$ and may be characterized by the observation of changes in these values. In this work, an attempt was made to capture the change in quantitative values in a clinically realistic context using the MYF, which is an indirect measure of the myelination degree of the brain, and the EPWF, which is an indirect measure of edema in the brain. Therefore, we believe that BPF, MYF, and EPWF are complementary measures to monitor the quantity and quality of the patient's brain in relation to intervention or progress of disease or aging.

In conclusion, a model is presented in which each MRI acquisition voxel in the brain is composed of a myelin partial volume, a cellular partial volume, a free water partial volume, and an excess parenchymal water partial volume. The magnetization vector

\section{REFERENCES}

1. Back SA, Riddle A, McClure MM. Maturation-dependent vulnerability of perinatal white matter in premature birth. Stroke (2007) 38:724-30. doi:10.1161/01. STR.0000254729.27386.05

2. Fields RD. White matter in learning, cognition and psychiatric disorders. Trends Neurosci (2008) 31:361-70. doi:10.1016/j.tins.2008.04.001

3. Miller DH, Barkhof F, Frank JA, Parker GJ, Thompson AJ. Measurement of atrophy in multiple sclerosis: pathological basis, methodological aspects and clinical relevance. Brain (2002) 125:1676-95. doi:10.1093/brain/awf177

4. Bakshi R, Thompson AJ, Rocca MA, Pelletier D, Dousset V, Barkhof F, et al. MRI in multiple sclerosis: current status and future prospects. Lancet Neurol (2008) 7:615-25. doi:10.1016/S1474-4422(08)70137-6

5. Hinman JD, Abraham CR. What's behind the decline? The role of white matter in brain aging. Neurochem Res (2007) 32:2023-31. doi:10.1007/s11064-0079341-X

6. Peters A. The effects of normal aging on myelin and nerve fibers: a review. $J$ Neurocytol (2002) 31:581-93. doi:10.1023/A:1024157522651

7. Matsusue E, Sugihara S, Fujii S, Ohama E, Kinoshita T, Ogawa T. White matter changes in elderly people: MR-pathologic correlations. Magn Reson Med Sci (2006) 5:99-104. doi:10.2463/mrms.5.99

8. Whittall KP, MacKay AL, Graeb DA, Nugent RA, Li DK, Paty DW. In vivo measurement of T2 distributions and water contents in normal human brain. Magn Reson Med (1997) 37:34-43. doi:10.1002/mrm.1910370107

9. Webb S, Munro CA, Midha R, Stanisz GJ. Is multicomponent T2 a good measure of myelin content in peripheral nerve? Magn Reson Med (2003) 49:638-45. doi:10.1002/mrm.10411

10. MacKay A, Laule C, Vavasour I, Bjarnason T, Kolind S, Mädler B. Insights into brain microstructure from the T2 distribution. Magn Reson Imaging (2006) 24:515-25. doi:10.1016/j.mri.2005.12.037

11. Bjarnason TA, Vavasour IM, Chia CL, MacKay AL. Characterization of the NMR behaviour of white matter in bovine brain. Magn Reson Med (2005) 54:1072-81. doi:10.1002/mrm.20680

12. Laule C, Leung E, Lis DK, Traboulsee AL, Paty DW, MacKay AL, et al. Myelin water imaging in multiple sclerosis: quantitative correlations with histopathology. Mult Scler (2006) 12:747-53. doi:10.1177/1352458506070928 evolution during an MRI quantification sequence was simulated for all partial volume distributions. The parameters of the model were obtained using spatially normalized brain data of a group of healthy control subjects. The differences for a pathological brain were described with myelin loss and the presence of excess parenchymal water. Application of the model showed clear differences between the control group and a spatially normalized MS group, as well as among three individual examples of highresolution imaging of a healthy middle-aged subject, an elderly control subject, and an MS patient. Using this model, clinically important information, such as the brain volume, degree of myelination, and degree of edema, may be estimated based on an acquisition with a clinically acceptable scan time.

\section{AUTHOR CONTRIBUTIONS}

MW, ME, and AT contributed to the design, the acquisition, analysis, and interpretation of data for the work; MW, ME, AT, and $\mathrm{PL}$ all contributed to writing, reviewing and given final approval of the manuscript.

\section{FUNDING}

The Linköping University and County Council Östergötland are acknowledged for funding this work.

13. Deoni SC, Rutt BK, Arun T, Pierpaoli C, Jones DK. Gleaning multicomponent $\mathrm{T} 1$ and T2 information from steady-state imaging data. Magn Reson Med (2008) 60:1372-87. doi: $10.1002 / \mathrm{mrm} .21704$

14. Deoni SC, Dean DC III, O’Muircheartaigh J, Dirks H, Jerskey BA. Investigating white matter development in infancy and early childhood using myelin water faction and relaxation time mapping. Neuroimage (2012) 63:1038-53. doi:10. 1016/j.neuroimage.2012.07.037

15. Kumar R, Delshad S, Woo MA, Macey PM, Harper RM. Age-related regional brain T2-relaxation changes in healthy adults. J Magn Reson Imaging (2012) 35:300-8. doi:10.1002/jmri.22831

16. Neema M, Stankiewicz J, Arora A, Dandamudi VS, Batt CE, Guss ZD, et al. T1- and T2-based MRI measures of diffuse gray matter and white matter damage in patients with multiple sclerosis. J Neuroimaging (2007) 17:16S-21S. doi:10.1111/j.1552-6569.2007.00131.x

17. Oh J, Cha S, Aiken AH, Han ET, Crane JC, Stainsby JA, et al. Quantitative apparent diffusion coefficients and $\mathrm{T} 2$ relaxation times in characterizing contrast enhancing brain tumors and regions of peritumoral edema. J Magn Reson Imaging (2005) 21:701-8. doi:10.1002/jmri.20335

18. Larsson HB, Frederiksen J, Petersen J, Nordenbo A, Zeeberg I, Henriksen O, et al. Assessment of demyelineation, edema and gliosis by in-vivo determination of T1 and T2 in the brain of patients with acute attack of multiple sclerosis. Magn Reson Med (1989) 11:337-8. doi:10.1002/mrm.1910110308

19. Vymazal J, Righini A, Brooks RA, Canesi M, Mariani C, Leonardi M, et al T1 and T2 in the brain of healthy subjects, patients with Parkinson's disease and patients with multiple system athrophy: relation to iron content. Radiology (1999) 211:489-95. doi:10.1148/radiology.211.2.r99ma53489

20. Warntjes JBM, Dahlqvist Leinhard O, West J, Lundberg P. Rapid magnetic resonance quantification on the brain: optimization for clinical usage. Magn Reson Med (2008) 60:320-9. doi:10.1002/mrm.21635

21. Engström M, Warntjes JBM, Tisell A, Landtblom AM, Lundberg P. Multiparametric representation of voxel-based quantitative magnetic resonance imaging. PLoS One (2014) 9:e111688. doi:10.1371/journal.pone.0111688

22. Levesque R, Pike GB. Characterizing healthy and diseased white matter using quantitative magnetisation transfer and multicomponent T2 relaxometry: a unified view via a four-pool model. Magn Reson Med (2009) 62:1487-96. doi: $10.1002 / \mathrm{mrm} .22131$ 
23. Deoni SCL, Matthews L, Kolind SH. One component? Two components? Three? The effect of including a nonexchanging "free" water component in multicomponent driven equilibrium single pulse observation of T1 and T2. Magn Reson Med (2013) 70:147-54. doi:10.1002/mrm.24429

24. Kurtzke JF. Rating neurologic impairment in multiple sclerosis: an expanded disability status scale (EDSS). Neurology (1983) 33:1444-52. doi:10.1212/WNL. 33.11.1444

25. Meeker W, Escobar L. Teaching about approximate confidence regions based on maximum likelihood estimation. Am Stat (1995) 49:48-53. doi:10.1080/ 00031305.1995.10476112

26. Warntjes JB, Engström M, Tisell A, Lundberg P. Brain characterization using normalised quantitative magnetic resonance imaging. PLoS One (2013) 8:e70864. doi:10.1371/journal.pone.0070864

27. Cedersund G, Roll J. Systems biology: model based evaluation and comparison of potential explanations for given biological data. FEBS J (2009) 276:903-22. doi:10.1111/j.1742-4658.2008.06845.x

28. Krauss W, Gunnarsson M, Andersson T, Thunberg P. Accuracy and reproducibility of a quantitative magnetic resonance imaging method for concurrent measurements of tissue relaxation times and proton density. Magn Reson Imaging (2015) 33:584-91. doi:10.1016/j.mri.2015.02.013

29. Deoni SCL, Rutt BK, Peters TM. High resolution T1 and T2 mapping of the brain in a clinically acceptable time with DESPOT1 and DESPOT2. Magn Reson Med (2005) 53:237-41. doi:10.1002/mrm.20314

30. Deichmann R. Fast high-resolution T1 mapping of the human brain. Magn Reson Med (2005) 54:20-7. doi:10.1002/mrm.20552

31. Ernst T, Kreis R, Ross BD. Absolute quantitation of water and metabolites in the human brain. 1: compartments and water. J Magn Reson B (1993) 102:1-8. doi:10.1006/jmrb.1993.1055

32. Neeb H, Ermer V, Stocker T, Shah NJ. Fast quantitative mapping of absolute water content with full brain coverage. Neuroimage (2008) 42:1094-109. doi: 10.1016/j.neuroimage.2008.03.060
33. Mezer A, Yeatman JD, Stikov N, Kay KN, Cho NJ, Dougherty RF, et al. Quantifying the local tissue volume and composition in individual brains with magnetic resonance imaging. Nat Med (2013) 19:1667-72. doi:10.1038/nm.3390

34. Abbas Z, Gras V, Möllenhoff K, Oros-Peusquens A-M, Shah NJ. Quantitative water content mapping at clinically relevant field strengths: a comparative study at $1.5 \mathrm{~T}$ and $3 \mathrm{~T}$. Neuroimage (2015) 106:404-13. doi:10.1016/j.neuroimage. 2014.11.017

35. Zhang J, Kolind SH, Laule C, MacKay AL. Comparison of myelin water fraction from multiecho T2 decay curve and steady-state methods. Magn Reson Med (2015) 73:223-32. doi:10.1002/mrm.25125

36. Oh J, Han ET, Pelletier D, Nelson SJ. Measurement of in vivo multi-component $\mathrm{T} 2$ relaxation times for brain tissue using multi-slice T2 prep at 1.5 and $3 \mathrm{~T}$. Magn Reson Imaging (2006) 24:33-43. doi:10.1016/j.mri.2005.10.016

37. Levesque IR, Chia CL, Pike GB. Reproducibility of in vivo magnetic resonance imaging-based measurement of myelin water. J Magn Reson Imaging (2010) 32:60-8. doi:10.1002/jmri.22170

38. Laule C, Vavasour IM, Moore GR, Oger J, Li DK, Paty DW, et al. Water content and myelin water fraction in multiple sclerosis. A T2 relaxation study. J Neurol (2004) 251:284-93. doi:10.1007/s00415-004-0306-6

Conflict of Interest Statement: The authors declare that the research was conducted in the absence of any commercial or financial relationships that could be construed as a potential conflict of interest.

Copyright (c) 2016 Warntjes, Engström, Tisell and Lundberg. This is an open-access article distributed under the terms of the Creative Commons Attribution License (CC $B Y)$. The use, distribution or reproduction in other forums is permitted, provided the original author(s) or licensor are credited and that the original publication in this journal is cited, in accordance with accepted academic practice. No use, distribution or reproduction is permitted which does not comply with these terms. 